\title{
First results from DAMA/LIBRA and the combined results with DAMA/NaI
}

\author{
R. Bernabei ${ }^{1,2, a}$, P. Belli ${ }^{2}$, F. Cappella ${ }^{3,4}$, R. Cerulli ${ }^{5}$, C.J. Dai ${ }^{6}$, A. d'Angelo ${ }^{3,4}$, H.L. He ${ }^{6}$, A. Incicchitti ${ }^{4}$, \\ H.H. Kuang ${ }^{6}$, J.M. Ma ${ }^{6}$, F. Montecchia ${ }^{1,2}$, F. Nozzoli ${ }^{1,2}$, D. Prosperi ${ }^{3,4}$, X.D. Sheng ${ }^{6}$, Z.P. Ye Ye, $^{6,7}$ \\ ${ }^{1}$ Dipartimento di Fisica, Università di Roma “Tor Vergata”, 00133 Rome, Italy \\ ${ }^{2}$ INFN, Sezione Roma "Tor Vergata", 00133 Rome, Italy \\ ${ }^{3}$ Dipartimento di Fisica, Università di Roma "La Sapienza", 00185 Rome, Italy \\ ${ }^{4}$ INFN, Sezione Roma, 00185 Rome, Italy \\ ${ }^{5}$ Laboratori Nazionali del Gran Sasso, INFN, 67010 Assergi, Italy \\ ${ }^{6}$ IHEP, Chinese Academy, P.O. Box 918/3 100039 Beijing, China \\ ${ }^{7}$ University of Jing Gangshan, Jiangxi, China
}

Received: 19 March 2008 / Revised: 2 May 2008 / Published online: 8 August 2008

(c) Springer-Verlag / Società Italiana di Fisica 2008

\begin{abstract}
The highly radiopure $\simeq 250 \mathrm{~kg} \mathrm{NaI(Tl)} \mathrm{DAMA/}$ LIBRA set-up is running at the Gran Sasso National Laboratory of the INFN. In this paper the first result obtained by exploiting the model independent annual modulation signature for Dark Matter (DM) particles is presented. It refers to an exposure of 0.53 ton $\times y$ r. The collected DAMA/LIBRA data satisfy all the many peculiarities of the DM annual modulation signature. Neither systematic effects nor side reactions able to account for the observed modulation amplitude and to contemporaneously satisfy all the several requirements of this DM signature are available. Considering the former DAMA/NaI and the present DAMA/LIBRA data all together (total exposure 0.82 ton $\times$ yr), the presence of Dark Matter particles in the galactic halo is supported, on the basis of the DM annual modulation signature, at $8.2 \sigma$ C.L.; in particular, in the energy interval (2-6) $\mathrm{keV}$, the modulation amplitude is $(0.0131 \pm 0.0016) \mathrm{cpd} / \mathrm{kg} / \mathrm{keV}$ and the phase and the period are well compatible with June $2^{\text {nd }}$ and one year, respectively.
\end{abstract}

\section{Introduction}

DAMA/LIBRA is part of the DAMA project, which is mainly based on the development and use of low background scintillators [1-56].

In particular, the former DAMA/NaI and the present DAMA/LIBRA set-ups have the main aim to perform a direct detection of Dark Matter (DM) particles in the galactic

\footnotetext{
a e-mail: rita.bernabei@ roma2.infn.it
}

halo through the model independent annual modulation signature (originally suggested in Refs. [57, 58]).

The former DAMA/NaI experiment [1-29] has achieved many competitive results on rare processes and, in particular, has pointed out a model independent evidence for the presence of DM particles in the galactic halo with high C.L.

In 1996-while running the DAMA/NaI set-up-DAMA proposed to INFN to develop and build a one ton set-up [59] to further investigate Dark Matter particles and other rare processes. Thus, a second generation $R \& D$ project was funded to develop new highly radiopure $\mathrm{NaI}(\mathrm{Tl})$ detectors towards the achievement of an intermediate step: the $\simeq 250 \mathrm{~kg}$ highly radiopure $\mathrm{NaI}(\mathrm{Tl})$ DAMA/LIBRA (Large sodium Iodide Bulk for RAre processes) set-up, which is now in data taking.

The exploitation of the annual modulation DM signature with highly radiopure $\mathrm{NaI}(\mathrm{Tl})$ as target material can permit to answer - by direct detection and in a way largely independent on the nature of the candidate and on the astrophysical, nuclear and particle Physics assumptions-the main question: "Are there Dark Matter (DM) particles in the galactic halo?"

In particular, the use of the highly radiopure DAMA/ LIBRA (and, previously, DAMA/NaI) NaI(Tl) scintillators as target-detectors offers many specific advantages thanks e.g. to the intrinsic radiopurity, to the large sensitivity to many of the DM candidates, of the interactions and of astrophysical, nuclear and particle Physics scenarios, to the granularity of the set-up, to the data taking up to the $\mathrm{MeV}$ scale (even though the optimization is made for the lowest energy region), to the full control of the running conditions, etc. 
Phenomenological properties of some basic interaction mechanisms induced by DM particles are discussed, for instance, in Refs. [11-13, 15-18, 57, 58, 60-109]. The DM annual modulation signature exploits the effect of the Earth revolution around the Sun on the number of events induced by DM particles in a suitable low background set-up placed deep underground. In particular, as a consequence of its annual revolution, the Earth should be crossed by a larger flux of DM particles around roughly June $2^{\text {nd }}$ (when its rotational velocity is summed to the one of the solar system with respect to the Galaxy) and by a smaller one around roughly December $2^{\text {nd }}$ (when the two velocities are subtracted) [11]. Thus, the contribution of the signal to the counting rate in the $k$-th energy interval can be written as (see e.g. Refs. [11, 12]):

$S_{k}=S_{0, k}+S_{m, k} \cos \omega\left(t-t_{0}\right)$,

where: (i) $S_{0, k}$ is the constant part of the signal; (ii) $S_{m, k}$ is the modulation amplitude; (iii) $\omega=\frac{2 \pi}{T}$ with period $T$; (iv) $t_{0}$ is the phase.

This annual modulation signature is very distinctive since a seasonal effect induced by DM particles must simultaneously satisfy all the following requirements: (1) the rate must contain a component modulated according to a cosine function; (2) with one year period; (3) with a phase roughly around June $2^{\text {nd }}$ in case of usually adopted halo models (slight variations may occur in case of presence of non thermalized DM components in the halo); (4) this modulation must be present only in a well-defined low energy range, where DM particles can induce signals; (5) it must be present only in those events where just a single detector, among all the available ones in the used set-up, actually "fires" (single-hit events), since the probability that DM particles experience multiple interactions is negligible; (6) the modulation amplitude in the region of maximal sensitivity has to be $\lesssim 7 \%$ in case of usually adopted halo distributions, but it may be significantly larger in case of some particular scenarios such as e.g. those of Refs. [68, 69]. To mimic such a signature spurious effects or side reactions should be able not only to account for the observed modulation amplitude but also to contemporaneously satisfy all the requirements of the signature; none of these has been found or suggested by anyone over more than a decade (see e.g. Refs. [11, 12], the references therein and later).

The corollary question: "Which are exactly the nature of the DM particle(s) detected by the annual modulation signature and the related astrophysical, nuclear and particle Physics scenarios?" requires subsequent model dependent corollary analyses as those performed e.g. in Refs. [11-18]. One should stress that it does not exist any approach to investigate the nature of the candidate in the direct and indirect DM searches which can offer these information independently on assumed astrophysical, nuclear and particle Physics scenarios.
As regards complementary information from accelerators, and most noticeably from LHC, the existence of some of the possible candidates could be tested at some extent; this will be very useful. However, it is worth noting that interesting DM candidates and scenarios for them exist, which are beyond the reach of that class of experiments (but potentially capable of determining the annual modulation effect).

The main goal of the DAMA/LIBRA experiment is to further study the presence of DM particles in the galactic halo pointed out by the former DAMA/NaI experiment [11, 12] exploiting the annual modulation signature and to get improved information on the corollary quests on the nature of the candidate particle(s) and on the related astrophysical, nuclear and particle physics models. Moreover, second order effects are planned to be investigated (see e.g. Refs. [12, 14]), and dedicated data takings will also allow the study of many other rare processes (as e.g. already performed with DAMA/NaI [19-29]) thanks to the peculiarity of the experimental set-up.

DAMA/LIBRA and the former DAMA/NaI are the only experiments effectively exploiting in all the aspects the DM annual modulation signature, and with highly radiopure $\mathrm{NaI}(\mathrm{Tl})$ detectors. It is worth noting that approaches based on many selections and handling procedures to "reject" the electromagnetic component of the counting rate would not offer any signature for Dark Matter particles. In fact, even under the assumption of an "ideal" rejection of the electromagnetic component, the neutrons and the internal end-range $\alpha$ 's induce signals indistinguishable from recoils; these events cannot be estimated and subtracted in reliable manner at the needed level of precision. Moreover, part or all the signal can have electromagnetic nature, depending on the candidate and on its interaction type. In a safe investigation of the DM annual modulation signature those data handlings cannot be applied e.g. since their statistical nature would affect the annual modulation analysis. On the other hand the exploitation of the DM annual modulation signature, as known, acts itself as an effective background rejection $[57,58]$.

In this paper some main features of the DAMA/LIBRA set-up [110] will be shortly summarized in Sect. 2. The model independent experimental results obtained by DAMA/LIBRA (exposure of 0.53 ton $\times$ yr collected over 4 annual cycles) and the combined ones with DAMA/NaI (exposure of 0.29 ton $\times$ yr collected over 7 annual cycles) are presented (total exposure of 0.82 ton $\times$ yr) in Sect. 3 . In Sect. 4 the quantitative investigation on possible systematic effects and side processes is discussed. The corollary model dependent analyses on the candidate particle(s) and astrophysical, nuclear and particle physics scenarios will be presented elsewhere in a dedicated publication; here in Appendix just few arguments are mentioned for some illustrative purposes. 


\section{The experimental set-up}

The DAMA/LIBRA set-up, its main features and radiopurity have been discussed in the devoted Ref. [110]. Here we just shortly summarize some information.

The installation of DAMA/LIBRA started in July 2002 after the dismounting of the former DAMA/NaI. The experimental site as well as many components of the installation itself have been implemented. All the procedures performed during the dismounting of DAMA/NaI and the installation of DAMA/LIBRA detectors have been carried out in high purity (HP) Nitrogen atmosphere.

The sensitive part of DAMA/LIBRA is made of 25 highly radiopure $\mathrm{NaI}(\mathrm{Tl})$ crystal scintillators in a 5-rows 5-columns matrix. The identification number of each detector (as it will be used later) is from the left (looking at the set-up from the door of inner barrack): 1 to 5 in the bottom (first) row, 6 to 10 in the second row and so on. Each $\mathrm{NaI}(\mathrm{Tl})$ detector has $9.70 \mathrm{~kg}$ mass and a size of $(10.2 \times 10.2 \times 25.4) \mathrm{cm}^{3}$. The bare crystals are enveloped in Tetratec-teflon foils and encapsulated in radiopure $\mathrm{OFHC} \mathrm{Cu}$ housing; $10 \mathrm{~cm}$ long special quartz light guides act also as optical windows on the two end faces of the crystals and are coupled to two low background photomultipliers (PMT). The threshold of each one of the two PMTs on a detector is set at single photoelectron level; their coincidence provides the trigger of the detector. The software energy threshold has been cautiously taken at $2 \mathrm{keV}$ electron equivalent (hereafter $\mathrm{keV}$ ). The measured light response is 5.5-7.5 photoelectrons $/ \mathrm{keV}$ depending on the detector. The detectors are housed in a low radioactivity sealed copper box installed in the center of a low-radioactivity $\mathrm{Cu} / \mathrm{Pb} / \mathrm{Cd}$-foils/polyethylene/paraffin shield; moreover, about $1 \mathrm{~m}$ concrete (made from the Gran Sasso rock material) almost fully surrounds (mostly outside the barrack) this passive shield, acting as a further neutron moderator. The copper box is maintained in HP Nitrogen atmosphere in slightly overpressure with respect to the external environment; it is part of the 3-levels sealing system which excludes the detectors from environmental air. The whole installation is air-conditioned and the temperature is continuously monitored and recorded; moreover, it is worth noting that the detectors have copper housings in direct contact with the multi-tons passive shield and its huge heat capacity $\left(\approx 10^{6} \mathrm{cal} /{ }^{\circ} \mathrm{C}\right.$ ) further assures a relevant stability of the detectors operating temperature (see also later).

Following the same strategy as DAMA/NaI, on the top of the shield a glove-box (also continuously maintained in the HP Nitrogen atmosphere) is directly connected to the inner $\mathrm{Cu}$ box, housing the detectors, through $\mathrm{Cu}$ pipes. The pipes are filled with low radioactivity $\mathrm{Cu}$ bars (covered by $10 \mathrm{~cm}$ of low radioactive $\mathrm{Cu}$ and $15 \mathrm{~cm}$ of low radioactive $\mathrm{Pb}$ ) which can be removed to allow the insertion of radioactive sources for calibrating the detectors in the same running condition, without any contact with external air. The glovebox is also equipped with a compensation chamber.

A hardware/software system to monitor the running conditions is operative and self-controlled computer processes automatically control several parameters and manage alarms. For the electronic chain, the data acquisition system and for all other details see Ref. [110].

The DAMA/LIBRA set-up, as the former DAMA/NaI, allows the recording both of the single-hit events (those events where just one detector of many actually fires) and of the multiple-hit events (those events where more than one detector fire).

The experiment take data up to the $\mathrm{MeV}$ scale despite the optimization is made for the lowest energy region. The linearity and the energy resolution of the detectors at low and high energy have been investigated using several sources as discussed in Ref. [110]. In particular, as regards the low energy region, calibrations down to the $3.2 \mathrm{keV} \mathrm{X}$ ray have been carried out. During the production runs periodical calibrations (every $\simeq 10$ days) are carried out with ${ }^{241}$ Am sources, introduced in the proximity of the detectors by source holders inserted in the $\mathrm{Cu}$ pipes mentioned above; the latter one is also continuously maintained in the $\mathrm{HP} \mathrm{Ni-}$ trogen atmosphere.

The energy threshold, the PMT gain, the electronic line stability are continuously verified and monitored during the data taking by the routine calibrations, by the position and energy resolution of internal lines [110] and by the study of the hardware rate behaviors with time.

The main procedures of the DAMA data taking for the investigation of DM particles annual modulation signature are: (1) the data taking of each annual cycle starts from autumn/winter (when $\cos \omega\left(t-t_{0}\right) \simeq 0$ ) towards summer (maximum expected); (2) the routine calibrations with radioactive sources are performed about each 10 days (collecting typically $\simeq 10^{4}-10^{5}$ events per keV), moreover regularly intrinsic calibration are carried out, etc. [110]; (3) the on-line monitoring of all the running parameters is continuously carried out with automatic alarm to operator if any would go out of allowed range.

\section{The model-independent experimental results}

As mentioned, DAMA/LIBRA started the first operations in March 2003. However, in order to allow the decay of medium half-life isotopes [110], the data taking for the investigation of the annual modulation signature, reported here, has been started on September 9, 2003. Moreover, it is worth noting that one of the more external detectors (identification number 16) has been put out of operation few months after installation because of a PMT break; since the dis-installation and re-installation of this detector would require the opening of the set-up, the installation of the room 
Table 1 DAMA/LIBRA annual cycles. There $\alpha=\left\langle\cos ^{2} \omega\left(t-t_{0}\right)\right\rangle$ is the mean value of the squared cosine and $\beta=\left\langle\cos \omega\left(t-t_{0}\right)\right\rangle$ is the mean value of the cosine (the averages are taken over the live time of the data taking and $t_{0}=152.5$ day, i.e. June $2^{\text {nd }}$ ); thus, $\alpha-\beta^{2}$ indicates the variance of the cosine (i.e. it is 0.5 for a full year of data taking). The exposed mass in these four annual cycles is $232.8 \mathrm{~kg}$; see text

\begin{tabular}{llll}
\hline Period & & Exposure $(\mathrm{kg} \times$ day $)$ & $\alpha-\beta^{2}$ \\
\hline DAMA/LIBRA-1 & Sept. 9, 2003 - July 21, 2004 & 51405 & 0.562 \\
DAMA/LIBRA-2 & July 21, 2004 - Oct. 28, 2005 & 52597 & 0.467 \\
DAMA/LIBRA-3 & Oct. 28, 2005 - July 18, 2006 & 39445 & 0.591 \\
DAMA/LIBRA-4 & July 19, 2006 - July 17, 2007 & 49377 & 0.541 \\
Total & & 192824 & 0.537 \\
& & $\simeq 0.53$ ton $\times$ yr
\end{tabular}

for doing it in HP Nitrogen atmosphere and some time of stop of the experiment, we have delayed this. The related procedure are planned to occur in 2008 when also an upgrade of the electronics will occur. Therefore, the exposed mass in the four annual cycles, presented here, is $232.8 \mathrm{~kg}$ for a total exposure of 0.53 ton $\times y \mathrm{y}$.

The only data treatment, which is performed on the raw data, is to eliminate obvious noise pulses (mainly PMT noise, Cherenkov light in the light guides and in the PMT windows, and afterglows) near the energy threshold in the single-hit events [110]; the number of such pulses sharply decreases when increasing the number of available photoelectrons. In particular, as mentioned, the DAMA/LIBRA detectors are seen by two PMTs working in coincidence and this already strongly reduces the noise near the energy threshold for the single-hit events (of interest for the detection of DM particles), while obviously noise is practically absent in the multiple-hit events, since the probability to have random coincidences is negligible $\left(<3 \times 10^{-6}\right)$. This rejection of the noise near energy threshold is based on the different time structures of the pulse profile of noise pulses (time decay of order of tens ns) and of the scintillation pulses (time decay of order of hundreds ns). The high number of photoelectrons $/ \mathrm{keV}$ assures a very good separation between the two populations, nevertheless stringent acceptance windows are used in order to assure full noise rejection near energy threshold; related acceptance window efficiencies are measured by devoted source calibrations. For a description of the used procedure and details see Ref. [110].

Detailed information about the four annual cycles by DAMA/LIBRA, considered here, is given in Table 1 . In these annual cycles about $4.4 \times 10^{7}$ events have also been collected for energy calibrations and about $6.0 \times 10^{7}$ events for the evaluation of the acceptance windows efficiency for noise rejection near energy threshold [110]. The periodical calibrations and, in particular, those related with the acceptance windows efficiency mainly affect the duty cycle of the experiment; in the present data taking it is of the same order as the one of DAMA/NaI, despite the larger number of involved detectors, thanks also to the improvements in the electronics and in the data acquisition (DAQ) system.

Figure 1 shows the cumulative (over the 4 data taking periods and over all the DAMA/LIBRA detectors) low-energy distribution of the single-hit scintillation events of interest for the investigations on DM particles.

In order to further investigate the presence of DM particles in the galactic halo, a model-independent investigation of the annual modulation signature has been carried out by exploiting the time behavior of the residual rates of the single-hit events in the lowest energy regions of the DAMA/LIBRA data. These residual rates are calculated from the measured rate of the single-hit events (obviously corrections for the overall efficiency and for the acquisition dead time are already applied) after subtracting the constant part: $\left\langle r_{i j k}-f l a t_{j k}\right\rangle_{j k}$. Here $r_{i j k}$ is the rate in the considered $i$-th time interval for the $j$-th detector in the $k$-th energy bin, while $f a_{j k}$ is the rate of the $j$-th detector in the $k$-th energy bin averaged over the cycles. The average is made on all the detectors ( $j$ index) and on all the $1 \mathrm{keV}$ bins ( $k$ index) which constitute the considered energy interval. The weighted mean of the residuals must obviously be zero over one cycle.

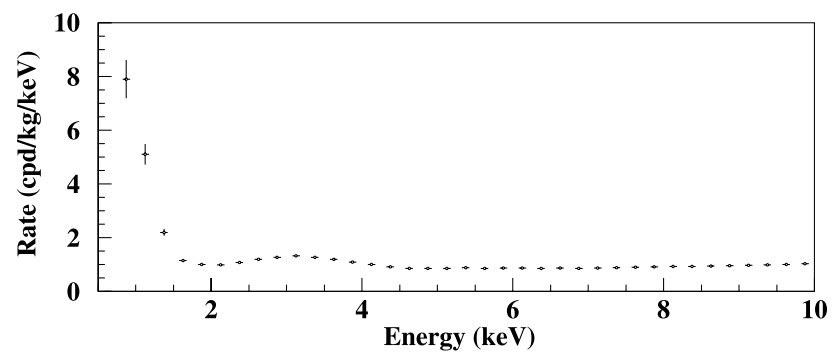

Fig. 1 Cumulative low-energy distribution of the single-hit scintillation events (that is each detector has all the others as veto), as measured by the DAMA/LIBRA detectors in an exposure of 0.53 ton $\times$ yr. The energy threshold of the experiment is $2 \mathrm{keV}$ and corrections for efficiencies are already applied 


\section{$2.4 \mathrm{keV}$}

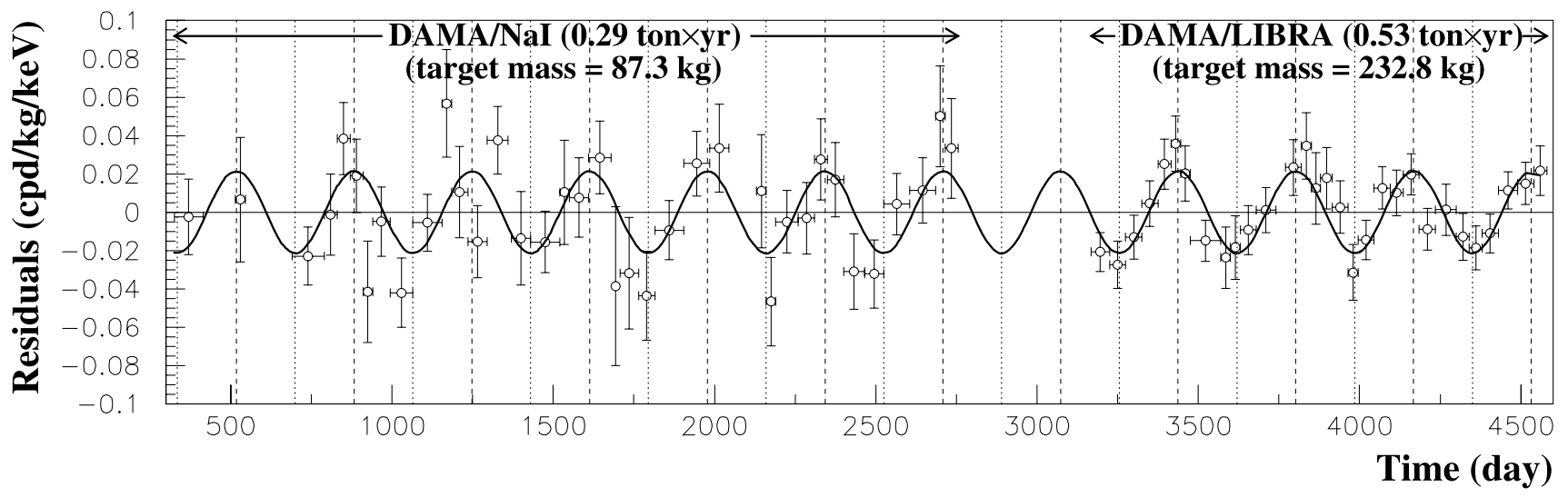

\section{$2.5 \mathrm{keV}$}
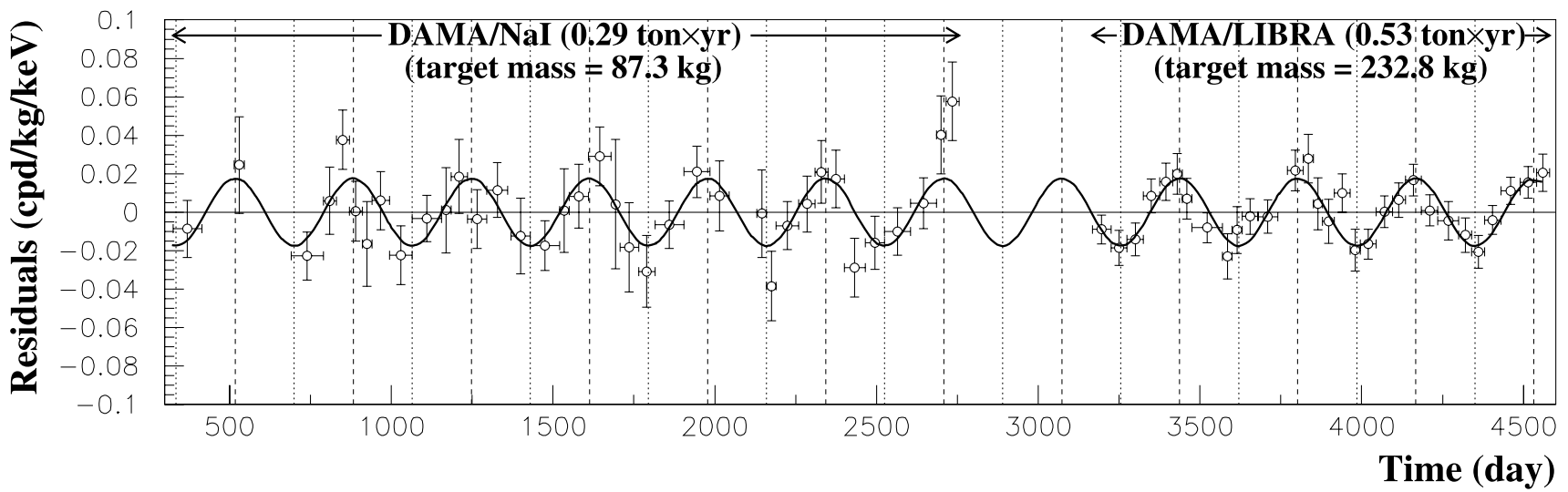

\section{$2-6 \mathrm{keV}$}

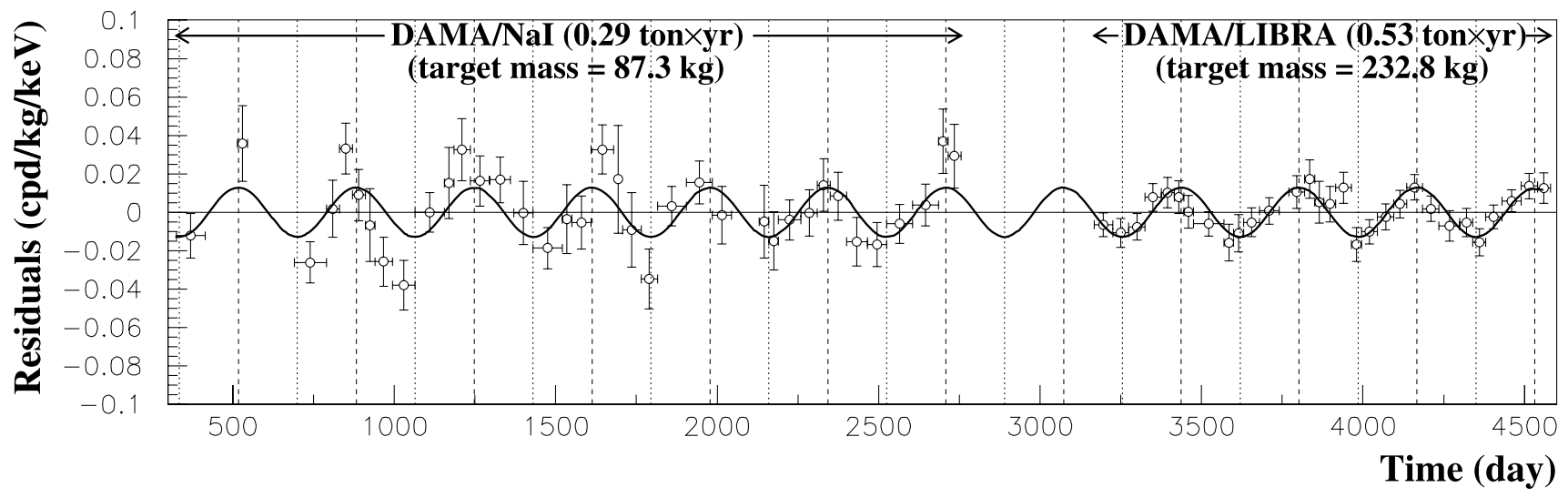

Fig. 2 Model-independent residual rate of the single-hit scintillation events, measured by the new DAMA/LIBRA experiment in the (2-4), (2-5) and (2-6) keV energy intervals as a function of the time. The residuals measured by DAMA/NaI and already published in Refs. [11, 12] are also shown. The zero of the time scale is January $1^{s t}$ of the first year of data taking of the former DAMA/NaI experiment. The experimental points present the errors as vertical bars and the associated time bin width as horizontal bars. The superimposed curves represent the cosinusoidal functions behaviors $A \cos \omega\left(t-t_{0}\right)$ with a period $T=\frac{2 \pi}{\omega}=1 \mathrm{yr}$, with a phase $t_{0}=152.5$ day (June $2^{\text {nd }}$ ) and with modulation amplitudes, $A$, equal to the central values obtained by best fit over the whole data, that is: $(0.0215 \pm 0.0026) \mathrm{cpd} / \mathrm{kg} / \mathrm{keV}$, $(0.0176 \pm 0.0020) \mathrm{cpd} / \mathrm{kg} / \mathrm{keV}$ and $(0.0129 \pm 0.0016) \mathrm{cpd} / \mathrm{kg} / \mathrm{keV}$ for the (2-4) keV, for the (2-5) keV and for the (2-6) keV energy intervals, respectively. See text. The dashed vertical lines correspond to the maximum of the signal (June $2^{\text {nd }}$ ), while the dotted vertical lines correspond to the minimum. The total exposure is 0.82 ton $\times \mathrm{yr}$ 
Table 2 Results obtained from the time behaviors of the residual rates of the single-hit scintillation events, collected by DAMA/NaI, by DAMA/LIBRA and by the two experiments all together in the (2-4),
(2-5) and (2-6) keV energy intervals. The data have been fitted with the function: $A \cos \omega\left(t-t_{0}\right)$. The last column shows the C.L. obtained from the fitted modulation amplitudes. See comments in the text

\begin{tabular}{|c|c|c|c|c|}
\hline & $A(\mathrm{cpd} / \mathrm{kg} / \mathrm{keV})$ & $T=\frac{2 \pi}{\omega}(\mathrm{yr})$ & $t_{0}$ (day) & C.L. \\
\hline \multicolumn{5}{|c|}{ DAMA/NaI } \\
\hline$(2-4) \mathrm{keV}$ & $0.0252 \pm 0.0050$ & $1.01 \pm 0.02$ & $125 \pm 30$ & $5.0 \sigma$ \\
\hline$(2-5) \mathrm{keV}$ & $0.0215 \pm 0.0039$ & $1.01 \pm 0.02$ & $140 \pm 30$ & $5.5 \sigma$ \\
\hline$(2-6) \mathrm{keV}$ & $0.0200 \pm 0.0032$ & $1.00 \pm 0.01$ & $140 \pm 22$ & $6.3 \sigma$ \\
\hline \multicolumn{5}{|c|}{ DAMA/LIBRA } \\
\hline$(2-4) \mathrm{keV}$ & $0.0213 \pm 0.0032$ & $0.997 \pm 0.002$ & $139 \pm 10$ & $6.7 \sigma$ \\
\hline$(2-5) \mathrm{keV}$ & $0.0165 \pm 0.0024$ & $0.998 \pm 0.002$ & $143 \pm 9$ & $6.9 \sigma$ \\
\hline$(2-6) \mathrm{keV}$ & $0.0107 \pm 0.0019$ & $0.998 \pm 0.003$ & $144 \pm 11$ & $5.6 \sigma$ \\
\hline \multicolumn{5}{|c|}{ DAMA/NaI + DAMA/LIBRA } \\
\hline$(2-4) \mathrm{keV}$ & $0.0223 \pm 0.0027$ & $0.996 \pm 0.002$ & $138 \pm 7$ & $8.3 \sigma$ \\
\hline$(2-5) \mathrm{keV}$ & $0.0178 \pm 0.0020$ & $0.998 \pm 0.002$ & $145 \pm 7$ & $8.9 \sigma$ \\
\hline$(2-6) \mathrm{keV}$ & $0.0131 \pm 0.0016$ & $0.998 \pm 0.003$ & $144 \pm 8$ & $8.2 \sigma$ \\
\hline
\end{tabular}

Figure 2 shows the time behavior (over three energy intervals) of the model-independent residual rates for singlehit events collected by the new DAMA/LIBRA experiment over four annual cycles $(0.53$ ton $\times$ yr). Those measured over seven annual cycles by the former DAMA/NaI experiment $[11,12](0.29$ ton $\times$ yr) are shown as well; the cumulative exposure of the two experiments is 0.82 ton $\times$ yr. The advantage of the increased exposed mass and exposure is evident.

In particular, the residual rates in the (2-4), (2-5) and (2-6) keV energy intervals are depicted in Fig. 2; the experimental points present the errors as vertical bars and the associated time bin width as horizontal bars. The superimposed curves represent the cosinusoidal functions behaviors $A \cos \omega\left(t-t_{0}\right)$ with a period $T=\frac{2 \pi}{\omega}=1 \mathrm{yr}$ and with a phase $t_{0}=152.5$ day (June $2^{\text {nd }}$ ) and modulation amplitudes, $A$, obtained by best fit over the whole data (DAMA/NaI \& DAMA/LIBRA). The dashed vertical lines correspond to the maximum of the signal (June $2^{\text {nd }}$ ), while the dotted vertical lines correspond to the minimum. We note that, for simplicity, in Fig. 2 the same time binning already considered e.g. in Refs. [11, 12] has been used. The result of this approach is similar by choosing other time binning, as it is also evident from the analysis on modulation amplitudes given in the following.

Table 2 summarizes the results obtained by fitting with the function $A \cos \omega\left(t-t_{0}\right)$ : (i) only the DAMA/NaI data $[11,12]$; (ii) only the DAMA/LIBRA data; (iii) all the data together. A clear modulation is present in all the energy intervals and the periods and phases agree with those expected in the case of a DM particle induced effect.

It is worthwhile remarking how the larger exposed mass per annual cycle has improved the fit; for example, the $\chi^{2} /$ d.o.f. of the best fit of the (2-6) $\mathrm{keV}$ single-hit residual rate from DAMA/NaI plus DAMA/LIBRA given in Table 2 is $53.2 / 64$. The period and phase substantially agree with $T=1 \mathrm{yr}$ and $t_{0}=152.5$ day.

We note that the difference in the (2-6) keV modulation amplitude between DAMA/NaI and DAMA/LIBRA depends mainly on the rate in the (5-6) $\mathrm{keV}$ energy bin. In particular, the modulation amplitudes for the (2-6) keV energy interval, obtained when fixing exactly the period at $1 \mathrm{yr}$ and the phase at 152.5 days, are $(0.019 \pm 0.003) \mathrm{cpd} / \mathrm{kg} / \mathrm{keV}$ and $(0.011 \pm 0.002) \mathrm{cpd} / \mathrm{kg} / \mathrm{keV}$ for DAMA/NaI and DAMA/LIBRA, respectively; thus, their difference is about $\simeq 2 \sigma$ which correspond to a modest, but non negligible probability. This is further supported by the analyses of the modulation amplitudes of each single year of DAMA/NaI and DAMA/LIBRA experiments, as reported in Fig. 3. There the central values obtained by best fit over the whole data set (see Fig. 2) are also depicted. The $\chi^{2}$ test $\left(\chi^{2}=4.9\right.$, 3.3 and 8.0 over 10 d.o.f. for the three energy intervals, respectively) and the run test (lower tail probabilities of $74 \%, 61 \%$ and $11 \%$ for the three energy intervals, respectively) accept the hypothesis at $90 \%$ C.L. that the modulation amplitudes are normally fluctuating around their best fit values. Thus, the cumulative result from DAMA/NaI and DAMA/LIBRA can be adopted.

In conclusion, the DAMA/LIBRA data are in substantial agreement with those of DAMA/NaI and the cumulative analysis favors the presence of a modulated cosine-like behavior at $8.2 \sigma$ C.L. (see Table 2). Moreover, the $\chi^{2}$ test on the residual rates disfavors the hypothesis of unmodulated behavior $(A=0)$ giving probabilities of $1.3 \times 10^{-4}$ $\left(\chi^{2} /\right.$ d.o.f. $\left.=117.7 / 67\right), 1.9 \times 10^{-4}\left(\chi^{2} /\right.$ d.o.f. $\left.=116.1 / 67\right)$ 

modulation amplitudes of each single annual cycle of

DAMA/NaI and DAMA/LIBRA experiments. The error bars are the related $1 \sigma$ errors. The same time scale and the same energy intervals as in Fig. 2 are adopted. The solid horizontal lines shows the central values obtained by best fit over the whole data set (see Fig. 2). The $\chi^{2}$ test and the run test accept the hypothesis at $90 \%$ C.L. that the modulation amplitudes are normally fluctuating around the best fit values. See text
Fig. 3 The data points are the
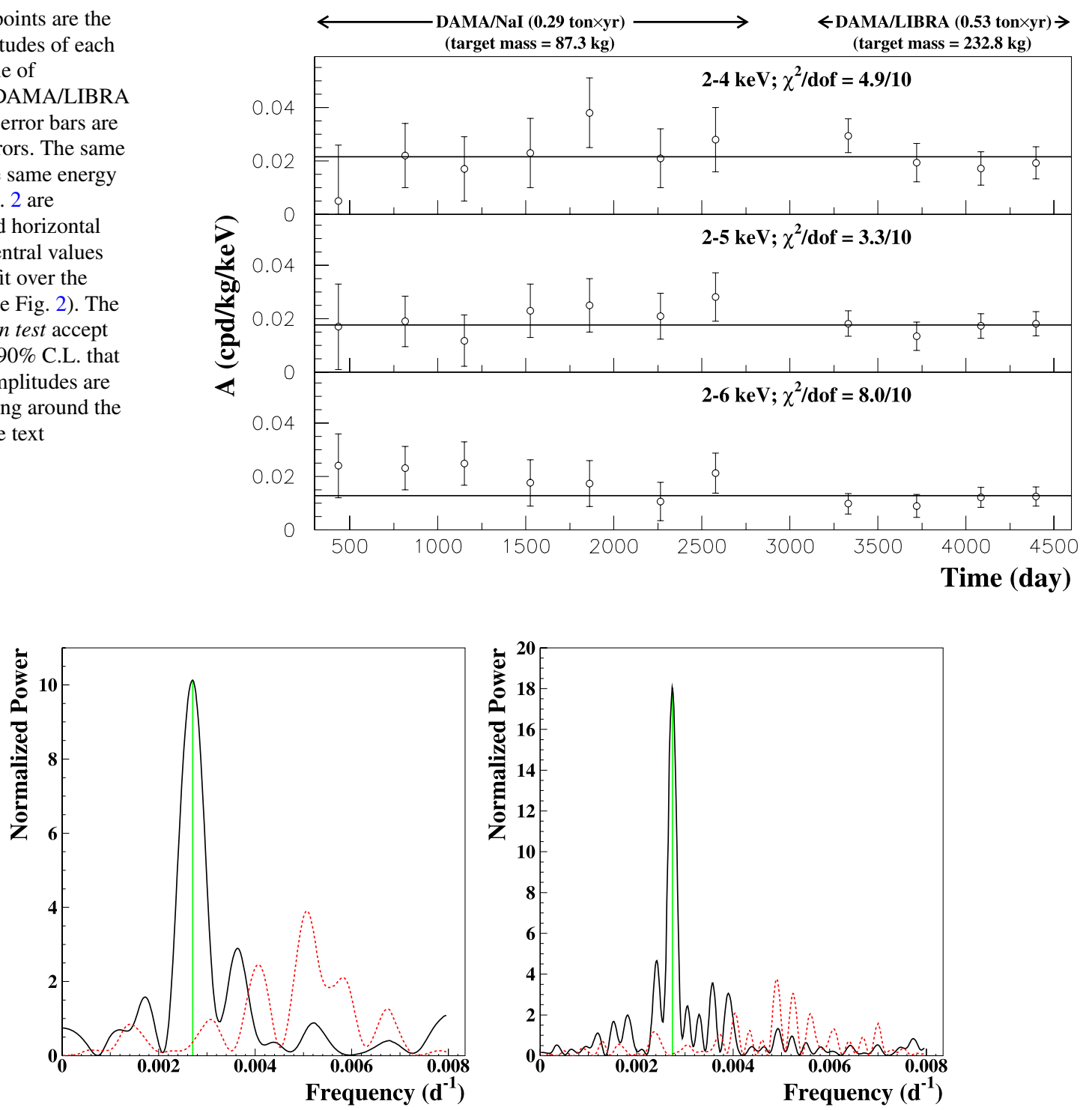

Fig. 4 Power spectrum of the measured single-hit residuals for the (2-6) $\mathrm{keV}$ (solid lines) and (6-14) keV (dotted lines) energy intervals calculated according to Refs. [111, 112], including also the treatment of the experimental errors and of the time binning. The data refer to: left-just to the DAMA/LIBRA data; right - to the cumulative DAMA/NaI and DAMA/LIBRA data; the case of DAMA/NaI has

and $1.8 \times 10^{-4}\left(\chi^{2} /\right.$ d.o.f. $\left.=116.4 / 67\right)$ for the three energy intervals, respectively.

The same data of Fig. 2 have also been investigated by a Fourier analysis (performed according to Refs. [111, 112] including also the treatment of the experimental errors and of the time binning); in particular, Fig. 4 shows the result for the DAMA/LIBRA and for the cumulative exposure; the one for DAMA/NaI alone has been given in Refs. [11, 12]. Here a clear peak for a period of 1 year is evident in the lowest energy interval (2-6) $\mathrm{keV}$.

In the same Fig. 4(left) there is also shown the absence of modulation in the region (6-14) $\mathrm{keV}$ just above the re-

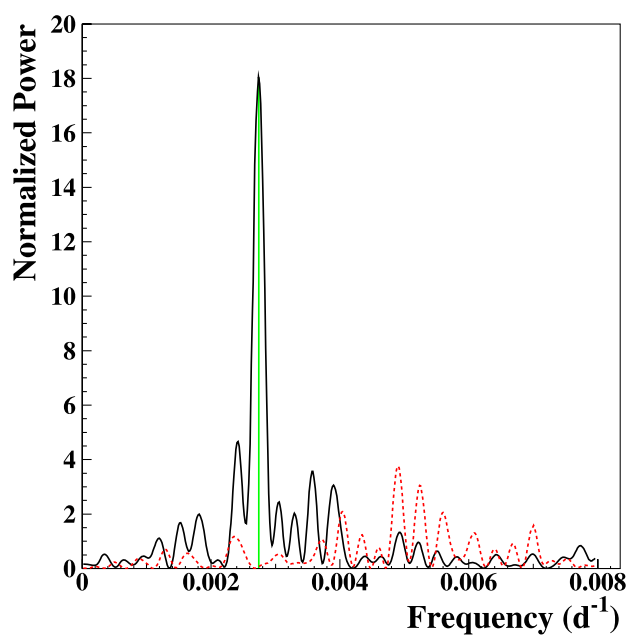

been given in Ref. $[11,12]$. As it can be seen, the principal mode present in the (2-6) $\mathrm{keV}$ energy interval corresponds to a frequency of $2.705 \times 10^{-3} \mathrm{~d}^{-1}$ and $2.737 \times 10^{-3} \mathrm{~d}^{-1}$, respectively (vertical lines); that is, they correspond to a period of $\simeq 1$ year. A similar peak is not present in the (6-14) $\mathrm{keV}$ energy interval just above

gion where the modulation is present in the DAMA/LIBRA data; in Fig. 4(right) the same is shown for the cumulative DAMA/NaI and DAMA/LIBRA data (for the DAMA/NaI data alone see Refs. [11, 12]).

Figure 5 compares the residuals in the (2-6) $\mathrm{keV}$ region and those in the (6-14) $\mathrm{keV}$ region just above for the DAMA/LIBRA data considered as collected in a single annual cycle. A clear modulation is present in the lowest energy interval, while it is absent just above. In fact, the best fitted modulation amplitude in the (6-14) keV energy region is well compatible with zero: $(0.0009 \pm 0.0011) \mathrm{cpd} / \mathrm{kg} / \mathrm{keV}$. 


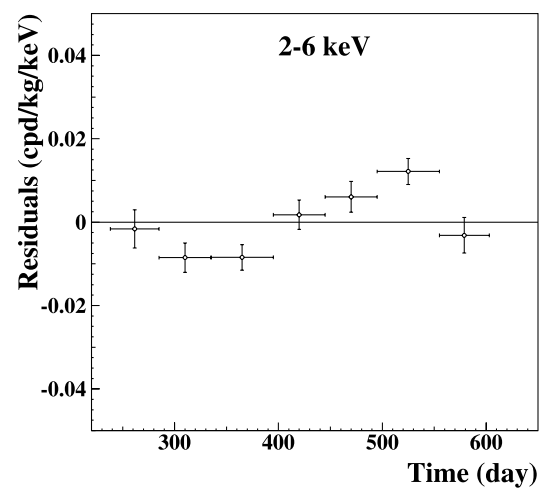

Fig. 5 Experimental residuals in the (2-6) $\mathrm{keV}$ region and those in the (6-14) $\mathrm{keV}$ region just above for the DAMA/LIBRA data considered as collected in a single annual cycle. The experimental points present the errors as vertical bars and the associated time bin width as horizon-

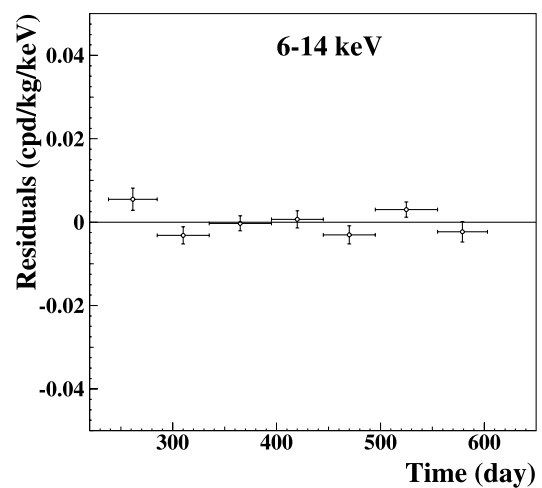

tal bars. The initial time is taken at August $7^{\text {th }}$. The clear modulation is present in the lowest energy interval, while it is absent just above. See text
Fig. 6 Experimental single-hit residual rates, as collected in a single annual cycle, for the total exposure of 0.82 ton $\times$ yr (i.e.

DAMA/NaI plus

DAMA/LIBRA) in the

(2-4) $\mathrm{keV}(\mathbf{a}),(2-5) \mathrm{keV}(\mathbf{b})$,

(2-6) $\mathrm{keV}(\mathbf{c})$ and

(6-14) $\mathrm{keV}$ (d) energy intervals.

The experimental points present the errors as vertical bars and the associated time bin width as horizontal bars. The initial time is taken at August $7^{\text {th }}$. Fitting the data with a cosinusoidal function when fixing exactly the period at $1 \mathrm{yr}$ and the phase at 152.5 days, the following amplitudes are obtained: (a) $(0.0204 \pm 0.0026) \mathrm{cpd} / \mathrm{kg} / \mathrm{keV}$; (b) $(0.0166 \pm 0.0020) \mathrm{cpd} / \mathrm{kg} / \mathrm{keV}$; (c) $(0.0125 \pm 0.0016) \mathrm{cpd} / \mathrm{kg} / \mathrm{keV}$; (d) $(0.0004 \pm 0.0010) \mathrm{cpd} / \mathrm{kg} / \mathrm{keV}$.

Thus, a clear modulation is present in the lowest energy regions, while it is absent just above a)
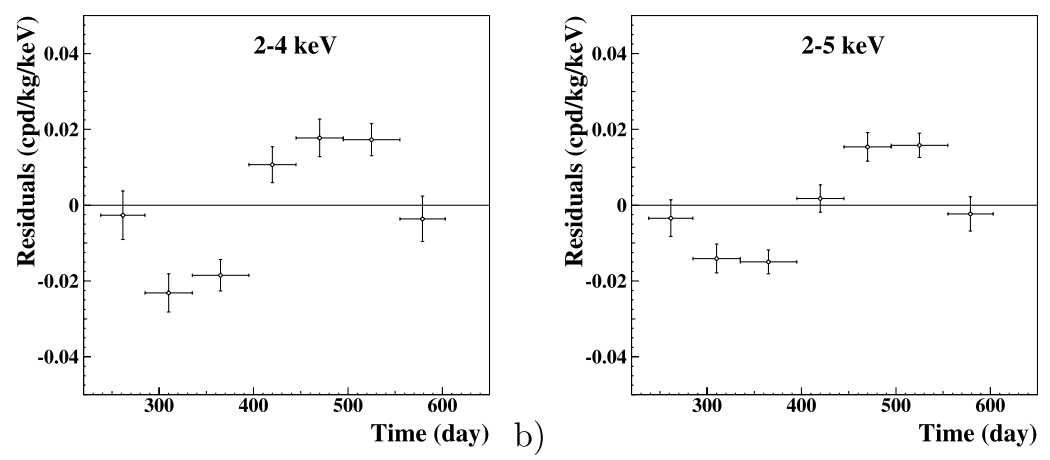

c)
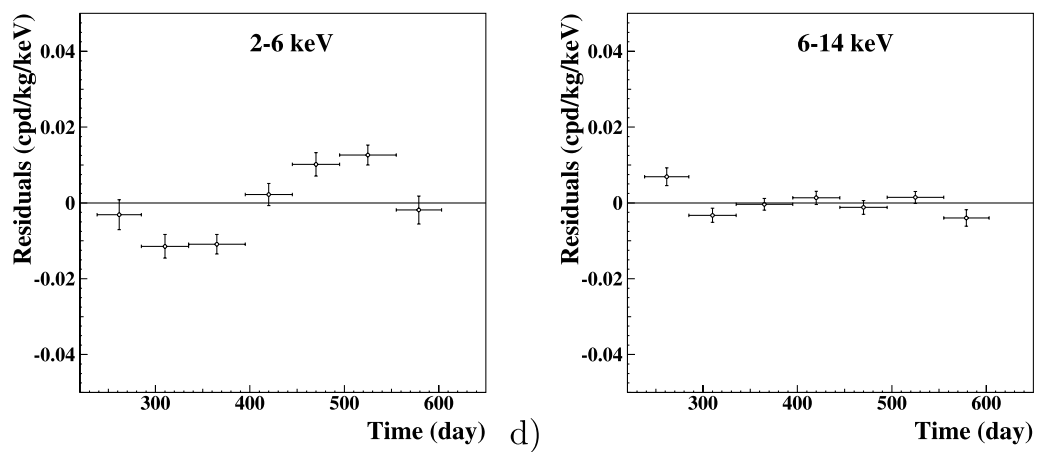

Finally, Fig. 6 shows-for various energy intervals-the experimental single-hit residual rates, as collected in a single annual cycle, for the total exposure of 0.82 ton $\times$ yr (i.e. DAMA/NaI \& DAMA/LIBRA).

To verify absence of annual modulation in other energy regions and, thus, to also verify the absence of any significant background modulation, the energy distribution measured during the data taking periods in energy regions not of interest for DM detection have been investigated. In fact, the background in the lowest energy region is essentially due to "Compton" electrons, X-rays and/or Auger electrons, muon induced events, etc., which are strictly correlated with the events in the higher energy part of the spectrum. Thus, if a modulation detected in the lowest energy region would be due to a modulation of the background (rather than to a signal), an equal or larger (sometimes much larger) modulation in the higher energy regions should be present. For this purpose, also in the present case we have investigated the rate integrated above $90 \mathrm{keV}, R_{90}$, as a function of the time. In Fig. 7 the distribution of the percentage variations of $R_{90}$ with respect to the mean values for all the detectors during the DAMA/LIBRA-1 to -4 annual cycles is given. It shows a cumulative Gaussian behavior with $\sigma \simeq 1 \%$, well accounted by the statistical spread expected from the used sampling 
time. This result excludes any significant background variation (see also later).

Moreover, fitting the time behavior of $R_{90}$ with phase and period as for DM particles, a modulation amplitude

Fig. 7 Distribution of the percentage variations of $R_{90}$ with respect to the mean values for all the detectors in the DAMA/LIBRA-1 to -4 annual cycles (histogram); the superimposed curve is a Gaussian fit. See text and Sect. 4

Fig. 8 Experimental residual rates over the four DAMA/LIBRA annual cycles for single-hit events (open circles) - class of events to which DM events belong-and for multiple-hits events (filled triangles) — class of events to which DM events do not belong - in the (2-4), (2-5) and (2-6) keV energy intervals. They have been obtained by considering for each class of events the data as collected in a single annual cycle and by using in both cases the same identical hardware and the same identical software procedures. The initial time of the scale is taken on August $7^{t h}$. The experimental points present the errors as vertical bars and the associated time bin width as horizontal bars. See text

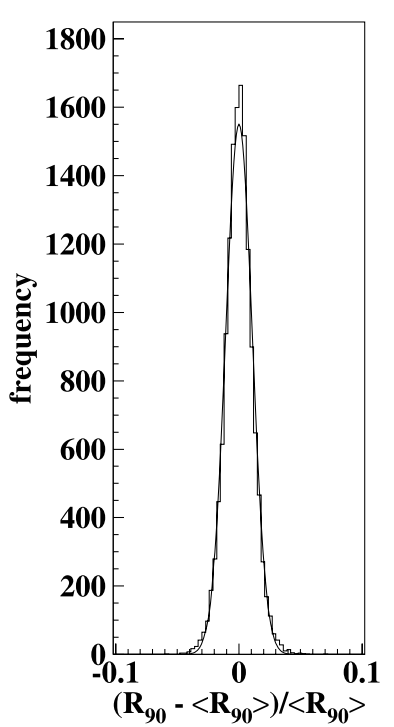

compatible with zero is found in each running period: $-(0.05 \pm 0.19) \mathrm{cpd} / \mathrm{kg},-(0.12 \pm 0.19) \mathrm{cpd} / \mathrm{kg},-(0.13 \pm$ $0.18) \mathrm{cpd} / \mathrm{kg}$, and $(0.15 \pm 0.17) \mathrm{cpd} / \mathrm{kg}$ for DAMA/LIBRA1 to -4 annual cycles, respectively. This excludes the presence of a background modulation in the whole energy spectrum at a level much lower than the effect found in the lowest energy region for the single-hit events; in fact, otherwiseconsidering the $R_{90}$ mean values-a modulation amplitude of order of tens $\mathrm{cpd} / \mathrm{kg}$, that $\simeq 100 \sigma$ far away from the measured value, would be present. Other arguments are also given in Sect. 4.

Finally, a further relevant investigation has been done by applying the same hardware and software procedures, used to acquire and to analyse the single-hit residual rate, to the multiple-hits one. In fact, since the probability that a DM particle interacts in more than one detector is negligible, a DM signal can be present just in the single-hit residual rate. Thus, this allows the test of the background behavior in the same energy interval of the observed positive effect. We remind that similar investigations have already been performed for the last two annual cycles of the DAMA/NaI experiment [12]. Thus, in Fig. 8 the residual rates of the singlehit events measured over the four DAMA/LIBRA annual
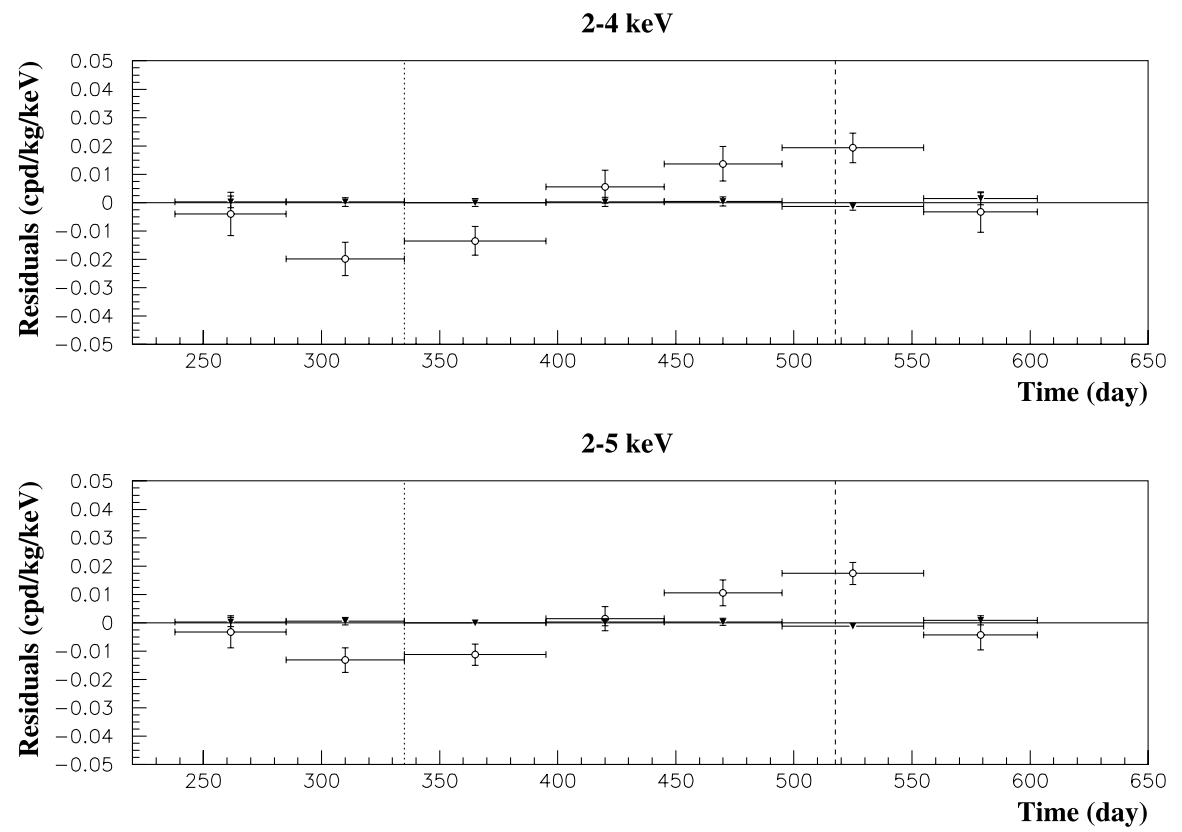

2-6 keV

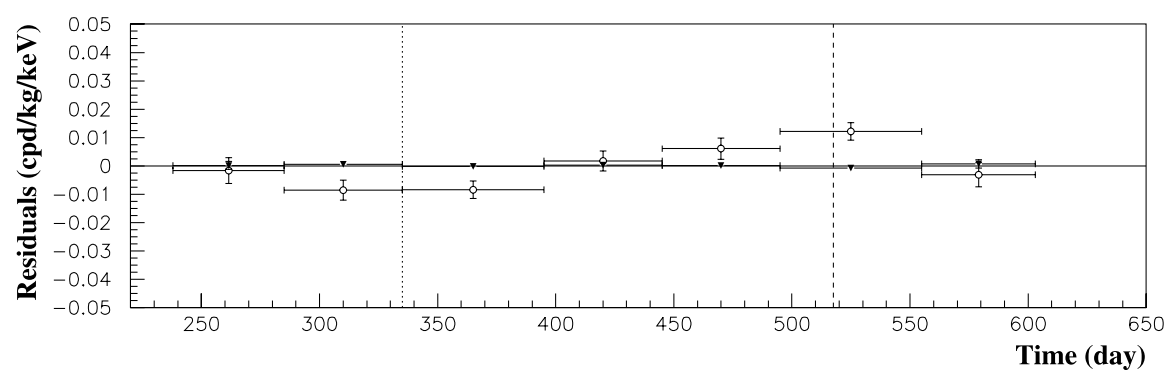




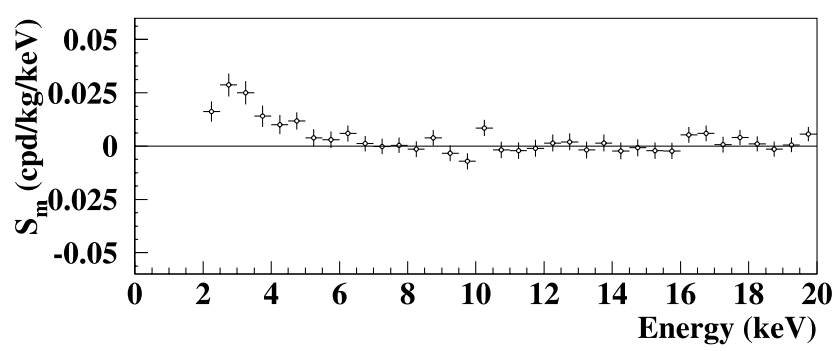

Fig. 9 Energy distribution of the $S_{m, k}$ variable for the total exposure (0.82 ton $\times$ yr, DAMA/NaI \& DAMA/LIBRA). See text. A clear modulation is present in the lowest energy region, while $S_{m, k}$ values compatible with zero are present just above. In fact, the $S_{m, k}$ values in the (6-20) keV energy interval have random fluctuations around zero with $\chi^{2}$ equal to 24.4 for 28 degrees of freedom. See also Appendix

cycles are reported, as collected in a single annual cycle, together with the residual rates of the multiple-hits events, in the considered energy intervals.

While, as already observed, a clear modulation is present in the single-hit events, the fitted modulation amplitudes for the multiple-hits residual rate are well compatible with zero: $-(0.0004 \pm 0.0008) \mathrm{cpd} / \mathrm{kg} / \mathrm{keV},-(0.0005 \pm$ $0.0007) \mathrm{cpd} / \mathrm{kg} / \mathrm{keV}$, and $-(0.0004 \pm 0.0006) \mathrm{cpd} / \mathrm{kg} / \mathrm{keV}$ in the energy regions (2-4), (2-5) and (2-6) $\mathrm{keV}$, respectively. Thus, again evidence of annual modulation with proper features as required by the DM annual modulation signature is present in the single-hit residuals (events class to which the DM particle induced events belong), while it is absent in the multiple-hits residual rate (event class to which only background events belong). Since the same identical hardware and the same identical software procedures have been used to analyse the two classes of events, the obtained result offers an additional strong support for the presence of a DM particle component in the galactic halo further excluding any side effect either from hardware or from software procedures or from background.

The annual modulation present at low energy can also be shown by depicting the $S_{m, k}$ values as a function of the energy; the $S_{m, k}$ is the modulation amplitude of the modulated part of the signal (see (1)) obtained by maximum likelihood method over the data considering $T=1 \mathrm{yr}$ and $t_{0}=152.5$ day. For such purpose the likelihood function of the single-hit experimental data in the $k$-th energy bin is defined as:

$\mathbf{L}_{\mathbf{k}}=\boldsymbol{\Pi}_{i j} e^{-\mu_{i j k}} \frac{\mu_{i j k}^{N_{i j k}}}{N_{i j k} !}$

where $N_{i j k}$ is the number of events collected in the $i$-th time interval (hereafter 1 day), by the $j$-th detector and in the $k$-th energy bin. $N_{i j k}$ follows a Poisson's distribution with expectation value $\mu_{i j k}=\left[b_{j k}+S_{0, k}+S_{m, k} \cdot \cos \omega\left(t_{i}-\right.\right.$ $\left.\left.t_{0}\right)\right] M_{j} \Delta t_{i} \Delta E \epsilon_{j k}$. The $b_{j k}$ are the background contributions, $M_{j}$ is the mass of the $j$-th detector, $\Delta t_{i}$ is the detector

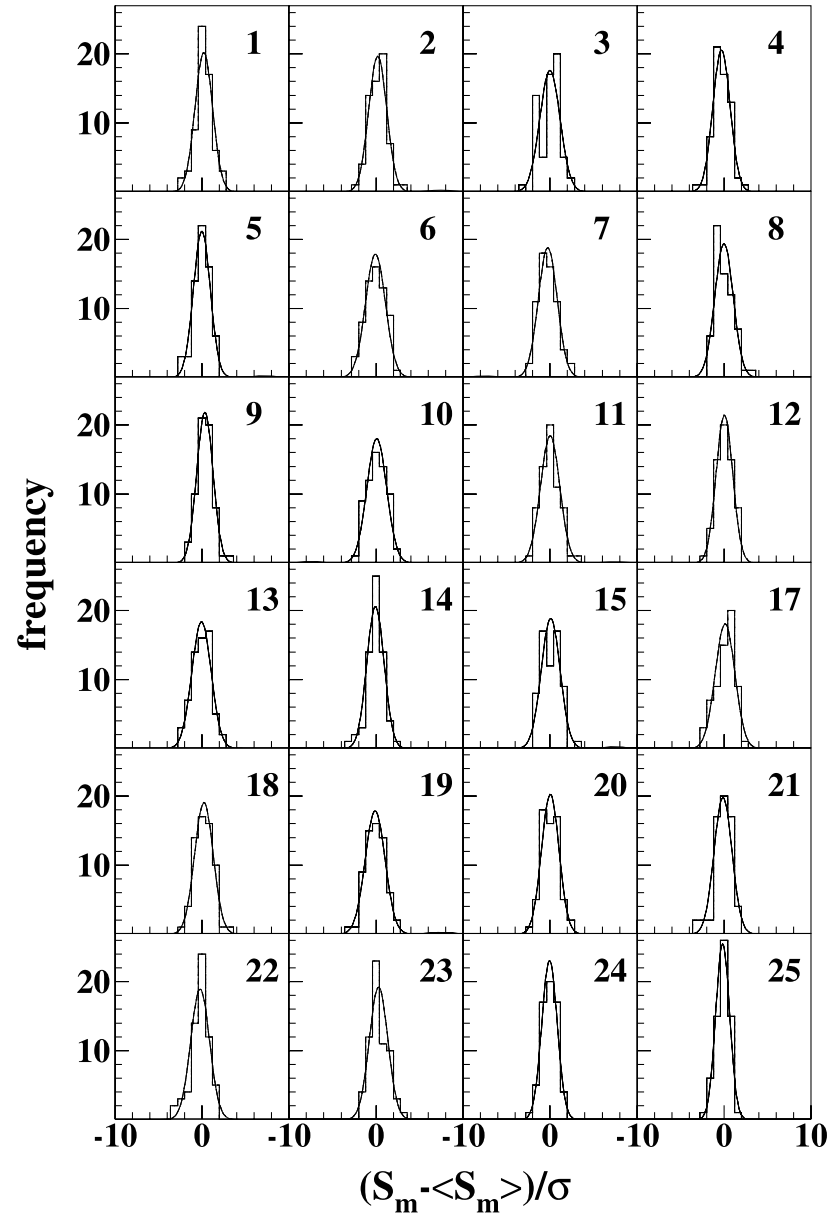

Fig. 10 Distributions (histograms) of the variable $\frac{S_{m}-\left\langle S_{m}\right\rangle}{\sigma}$ where $\sigma$ are the errors associated to the $S_{m}$ values and $\left\langle S_{m}\right\rangle$ are the mean values of the modulation amplitudes averaged over the detectors and the annual cycles for each considered energy bin (here $\Delta E=0.25 \mathrm{keV}$ ). The entries of each histogram are 64 (16 energy bins in the (2-6) keV energy interval and 4 DAMA/LIBRA annual cycles); the r.m.s. values are reported in Fig. 11(bottom). The superimposed curves are Gaussian fits. Each panel refers to a single DAMA/LIBRA detector (the identification number is quoted; the detector 16 was out of trigger, see above)

running time during the $i$-th time interval, $\Delta E$ is the chosen energy bin, $\epsilon_{j k}$ is the overall efficiency. The usual procedure is to minimize the function $y_{k}=-2 \ln \left(\mathbf{L}_{\mathbf{k}}\right)-$ const for each energy bin; the free parameters of the fit are the $\left(b_{j k}+S_{0, k}\right)$ contributions and the $S_{m, k}$ parameter.

In Fig. 9 the $S_{m, k}$ are reported for the seven annual cycles of DAMA/NaI and for the four annual cycles of DAMA/LIBRA in each considered energy bin (here $\Delta E=$ $0.5 \mathrm{keV})$. It can be inferred that positive signal is present in the (2-6) keV energy interval, while $S_{m, k}$ values compatible with zero are present just above. In fact, the $S_{m, k}$ values in the (6-20) keV energy interval have random fluctuations around zero with $\chi^{2}$ equal to 24.4 for 28 degrees of freedom. All this confirms the previous analyses. 

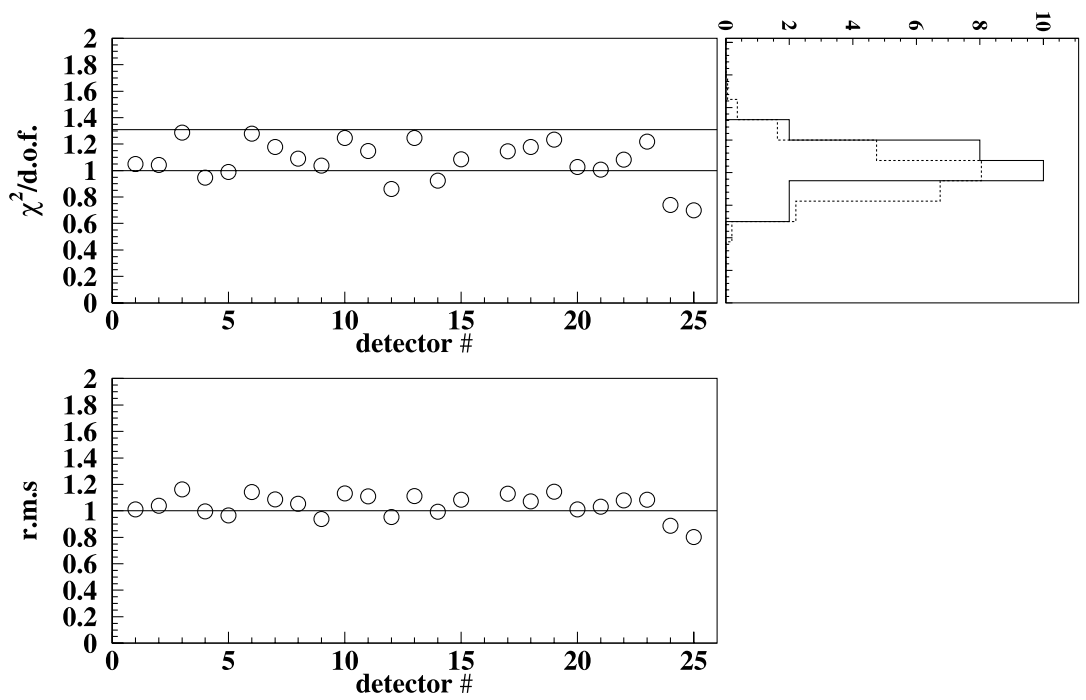

Fig. 11 Top left: $\chi^{2} /$ d.o.f. values of $S_{m}$ distributions around their mean value for each DAMA/LIBRA detector in the (2-6) keV energy interval for the four annual cycles. The line at $\chi^{2} /$ d.o.f. $=1.31$ corresponds to an upper tail probability of $5 \%$. All the $\chi^{2} /$ d.o.f. values are below this line and, thus, at $95 \%$ C.L. the observed annual modulation effect is well distributed in all the detectors. Top right: distribution of the twenty-four points in the top left panel (solid histogram) compared with the $\chi^{2}$ distribution with 64 degrees of freedom; the comparison gives a $\chi^{2} /$ d.o.f. $=8.1 / 7$. See text. Bottom: standard deviations of the $x$ variable for the DAMA/LIBRA detectors; see also Fig. 10. See text
The method also allows the extraction of the $S_{m}$ (hereafter the index $k$ is omitted) values for each detector, for each annual cycle as well as for each considered energy bin. The $S_{m}$ are expected to follow a normal distribution in absence of any systematic effects. Therefore, in order to show if they are statistically well distributed in all the crystals, in all the annual cycles and in the energy bins, the variable $x=\frac{S_{m}-\left\langle S_{m}\right\rangle}{\sigma}$ is considered. Here, $\sigma$ are the errors associated to $S_{m}$ and $\left\langle S_{m}\right\rangle$ are the mean values of the $S_{m}$ averaged over the detectors and the annual cycles for each considered energy bin (in the following $\Delta E=0.25 \mathrm{keV}$ ). Similar investigations have already been performed also for DAMA/NaI [11, 12].

Figure 10 shows the distributions of the variable $x$ for the DAMA/LIBRA data in the (2-6) $\mathrm{keV}$ energy interval plotted for each detector separately (i.e. the entries of each histogram are the $64 x$ values, evaluated for the 16 energy bins in the considered (2-6) keV energy interval and for the 4 DAMA/LIBRA annual cycles). These distributions allow one to conclude that the observed annual modulation effect is well distributed in all the detectors and annual cycles. In fact, the standard deviations of the $x$ variable for the DAMA/LIBRA detectors range from 0.80 to 1.16 (see also Fig. 11(bottom)). Defining $\chi^{2}=\Sigma x^{2}$-where the sum is extended over all the $64 x$ values $-\chi^{2} /$ d.o.f. values ranging from 0.7 to 1.28 (see Fig. 11(top)) are obtained. The corresponding upper tail probabilities range from about $97 \%$ to $6 \%$. Therefore, the observed annual modulation effect is well distributed in all the detectors at $95 \%$ C.L. The $\chi^{2} /$ d.o.f. values of the DAMA/LIBRA detectors show a distribution around their expectation value (see Fig. 11(top)).
The twenty-four points follow a $\chi^{2}$ distribution with 64 degrees of freedom; in fact, when compared with the expectation in Fig. 11(top-right), a $\chi^{2} /$ d.o.f. $=8.1 / 7$ is obtained. The mean value of the twenty-four $\chi^{2} /$ d.o.f. values is 1.072 , slightly larger than expected. Although this can be still ascribed to statistical fluctuations (see before), let us ascribe it to a possible systematics. In this case, one would have an additional error of $\leq 5 \times 10^{-4} \mathrm{cpd} / \mathrm{kg} / \mathrm{keV}$, if quadratically combined, or $\leq 7 \times 10^{-5} \mathrm{cpd} / \mathrm{kg} / \mathrm{keV}$, if linearly combined, to the modulation amplitude measured in the (2-6) $\mathrm{keV}$ energy interval. This possible additional error $-\leq 4.7 \%$ or $\leq 0.7 \%$, respectively, of the DAMA/LIBRA modulation amplitude — can be considered as an upper limit of possible systematic effects (see also later the dedicated section).

The above arguments demonstrate that the modulation amplitudes are statistically well distributed in all the crystals, in all the data taking periods and in the considered energy bins.

Among further additional tests, which have been carried out, we mention the analysis of the modulation amplitudes as a function of the energy (similarly as in Fig. 9) separately for the nine inner detectors and the fifteen external ones. The obtained values are fully in agreement. The hypothesis that the two sets of modulation amplitudes as a function of the energy belong to same distribution has been verified by $\chi^{2}$ test. It gives $\chi^{2} /$ d.o.f. $=6.9 / 8$ and $12.4 / 16$ for the energy intervals (2-4) and (2-6) keV, respectively. This shows that the effect is also well shared between inner and external detectors.

Let us, finally, release the assumption of a phase $t_{0}=$ 152.5 day in the maximum likelihood procedure to evaluate 
Fig. $122 \sigma$ contours in the plane $\left(S_{m}, Z_{m}\right)$ (left) and in the plane $\left(Y_{m}, t^{*}\right)($ right $)$ for the (2-6) $\mathrm{keV}$ and (6-14) $\mathrm{keV}$ energy intervals. The contours have been obtained by the maximum likelihood method, considering the seven annual cycles of DAMA/NaI and the four annual cycles of DAMA/LIBRA all together

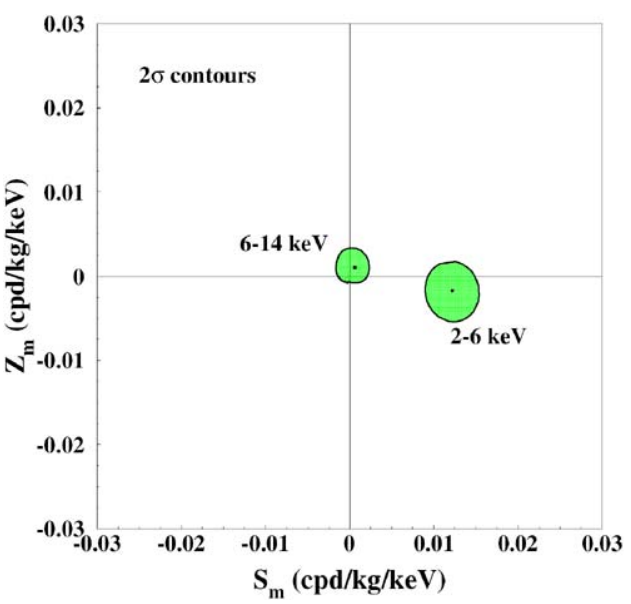

the modulation amplitudes, as performed above; that is, let us alternatively write the expectation values, $\mu_{i j k}$, in (2) as:

$$
\begin{aligned}
\mu_{i j k}= & {\left[b_{j k}+S_{0, k}+S_{m, k} \cos \omega\left(t_{i}-t_{0}\right)\right.} \\
& \left.+Z_{m, k} \sin \omega\left(t_{i}-t_{0}\right)\right] M_{j} \Delta t_{i} \Delta E \epsilon_{j k} \\
= & {\left[b_{j k}+S_{0, k}+Y_{m, k} \cos \omega\left(t_{i}-t^{*}\right)\right] M_{j} \Delta t_{i} \Delta E \epsilon_{j k} . }
\end{aligned}
$$

Obviously, for signals induced by DM particles one would expect: (i) $Z_{m, k} \sim 0$ (because of the orthogonality between the cosine and the sine functions); (ii) $S_{m, k} \simeq Y_{m, k}$; (iii) $t^{*} \simeq t_{0}=152.5$ day. In fact, these conditions hold for most of the dark halo models; however, it is worth noting that slight differences can be expected in case of possible contributions from non-thermalized DM components, such as e.g. the SagDEG stream [14] and the caustics [113]. The analysis has been performed considering the data of the seven annual cycles of DAMA/NaI and the four annual cycles of DAMA/LIBRA all together. Figure 12(left) shows the $2 \sigma$ contours in the plane $\left(S_{m}, Z_{m}\right)$ for the $(2-6) \mathrm{keV}$ and (6-14) keV energy intervals and Fig. 12(right) shows, instead, those in the plane $\left(Y_{m}, t^{*}\right)$. The best fit values for the (2-6) $\mathrm{keV}$ energy interval are ( $1 \sigma$ errors): $S_{m}=(0.0122 \pm 0.0016) \mathrm{cpd} / \mathrm{kg} / \mathrm{keV} ; Z_{m}=-(0.0019 \pm$ $0.0017) \mathrm{cpd} / \mathrm{kg} / \mathrm{keV} ; Y_{m}=(0.0123 \pm 0.0016) \mathrm{cpd} / \mathrm{kg} / \mathrm{keV}$; $t^{*}=(144.0 \pm 7.5)$ day; while for the $(6-14) \mathrm{keV}$ energy interval are: $S_{m}=(0.0005 \pm 0.0010) \mathrm{cpd} / \mathrm{kg} / \mathrm{keV}$; $Z_{m}=(0.0011 \pm 0.0012) \mathrm{cpd} / \mathrm{kg} / \mathrm{keV} ; \quad Y_{m}=(0.0012 \pm$ $0.0011) \mathrm{cpd} / \mathrm{kg} / \mathrm{keV}$ and $t^{*}$ obviously not determined (see Fig. 12). These results confirm those achieved above by analysing the residuals. In particular, a modulation amplitude is present in the lower energy intervals and the period and the phase agree with those expected for DM induced signals. Finally, forcing to zero the contribution of the cosine function in (3), the $Z_{m, k}$ values as function of the energy have also been determined by using the same procedure. $\mathrm{Ob}-$ viously, such values are expected to be zero in case of presence of a DM particles' signal with $t_{0}=152.5$ day. From the data we get: $-(0.0046 \pm 0.0041) \mathrm{cpd} / \mathrm{kg} / \mathrm{keV},-(0.0036 \pm$ $0.0042) \mathrm{cpd} / \mathrm{kg} / \mathrm{keV},(0.0036 \pm 0.0034) \mathrm{cpd} / \mathrm{kg} / \mathrm{keV}$, and $-(0.0015 \pm 0.0032) \mathrm{cpd} / \mathrm{kg} / \mathrm{keV}$, for (2-3), (3-4), (4-5), (5-6) energy intervals, respectively. Moreover, the $\chi^{2}$ test applied to the data in the $(2-14) \mathrm{keV}$ energy region $\left(\chi^{2}\right.$ equal to 12.4 for 12 degrees of freedom) supports the hypothesis that the $Z_{m, k}$ values are simply fluctuating around zero.

In conclusion, an annual modulation satisfying all the requirements of the DM annual modulation signature has been observed also in DAMA/LIBRA with high C.L.; it credits cumulatively with DAMA/NaI an evidence for the presence of DM particles in the galactic halo at $8.2 \sigma$ C.L.

\section{Further investigation on possible systematic effects and side reactions}

Also the data of the first four annual cycles of DAMA/ LIBRA as previously those of DAMA/NaI fulfill the requirements of the DM annual modulation signature and preliminary investigation on absence of any significant systematics or side reaction effect is already present in some parts of the previous section; however, here the argument will be addressed in more details.

Sometimes naive statements are put forwards as the fact that in nature several phenomena may show annual periodicity. It is worth noting that the point is whether they might mimic the annual modulation signature in DAMA/LIBRA, i.e. whether they might be not only quantitatively able to account for the observed modulation amplitude but also able to contemporaneously satisfy all the requirements of the DM annual modulation signature. This was deeply investigated in the former DAMA/NaI experiment (see e.g. Refs. [11, 12] and references therein; no one has been either found or suggested by anyone so far) and will be further addressed in the following for the present DAMA/LIBRA data. 
Table 3 Modulation amplitudes ( $1 \sigma$ error) obtained by fitting the time behaviors of the main running parameters including a possible annual modulation with phase and period as for DM particles. These running parameters, acquired with the production data, are: (i) the operating temperature of the detectors; (ii) the HP Nitrogen flux in the inner $\mathrm{Cu}$ box housing the detectors; (iii) the pressure of the $\mathrm{HP} \mathrm{Ni}$ - trogen atmosphere of the inner $\mathrm{Cu}$ box housing the detectors; (iv) the environmental Radon in the inner part of the barrack from which the detectors are however excluded (see text and Ref. [110] for details); (v) the hardware rate above single photoelectron threshold. All the measured amplitudes are compatible with zero

\begin{tabular}{lllll}
\hline & DAMA/LIBRA-1 & DAMA/LIBRA-2 & DAMA/LIBRA-3 & DAMA/LIBRA-4 \\
\hline Temperature & $-(0.0001 \pm 0.0061)^{\circ} \mathrm{C}$ & $(0.0026 \pm 0.0086)^{\circ} \mathrm{C}$ & $(0.001 \pm 0.015)^{\circ} \mathrm{C}$ & $(0.0004 \pm 0.0047)^{\circ} \mathrm{C}$ \\
Flux & $(0.13 \pm 0.22) \mathrm{l} / \mathrm{h}$ & $(0.10 \pm 0.25) \mathrm{l} / \mathrm{h}$ & $-(0.07 \pm 0.18) \mathrm{l} / \mathrm{h}$ & $-(0.05 \pm 0.24) \mathrm{l} / \mathrm{h}$ \\
Pressure & $(15 \pm 30) 10^{-3} \mathrm{mbar}$ & $-(13 \pm 25) 10^{-3} \mathrm{mbar}$ & $(22 \pm 27) 10^{-3} \mathrm{mbar}$ & $(1.8 \pm 7.4) 10^{-3} \mathrm{mbar}$ \\
Radon & $-(0.029 \pm 0.029) \mathrm{Bq} / \mathrm{m}^{3}$ & $-(0.030 \pm 0.027) \mathrm{Bq} / \mathrm{m}^{3}$ & $(0.015 \pm 0.029) \mathrm{Bq} / \mathrm{m}^{3}$ & $-(0.052 \pm 0.039) \mathrm{Bq} / \mathrm{m}^{3}$ \\
Hardware rate & $-(0.20 \pm 0.18) 10^{-2} \mathrm{~Hz}$ & $(0.09 \pm 0.17) 10^{-2} \mathrm{~Hz}$ & $-(0.03 \pm 0.20) 10^{-2} \mathrm{~Hz}$ & $(0.15 \pm 0.15) 10^{-2} \mathrm{~Hz}$ \\
\hline
\end{tabular}

Firstly, in order to continuously monitor the running conditions, several pieces of information are acquired with the production data and quantitatively analysed; note that information on technical aspects of DAMA/LIBRA has been discussed in Ref. [110], where the sources of possible residual radioactivity have also been analysed.

In particular, all the time behaviors of the running parameters, acquired with the production data, have been investigated. Table 3 shows the modulation amplitudes obtained for each annual cycle when fitting the time behaviors of the values of the main parameters including a cosine modulation with the same phase and period as for DM particles. As can be seen, all the measured amplitudes are well compatible with zero.

Let us now enter in more details.

\subsection{The temperature}

Since temperature at sea level varies along the year, sometimes it has been naively suggested that it can mimic the observed effect.

It is worth noting that the full experiment is placed underground and works in an air-conditioned environment; moreover, the detectors have $\mathrm{Cu}$ housing in direct contact with the multi-tons metallic passive shield whose huge heat capacity definitively assures a relevant stability of the detectors' operating temperature [110]. Nevertheless the operating temperature is read out by a probe and stored with the production data, in order to offer the possibility of quantitative investigations (see also above).

Specific information on the DAMA/LIBRA-1 to -4 annual cycles can be derived from Fig. 13; no evidence of any operating temperature modulation has been observed, as also quantitatively reported in Table 3 .

However, to properly evaluate the real effect of possible variations of the detectors' operating temperature on the
Fig. 13 Distribution of the relative variations of the operating temperature measured during the DAMA/LIBRA-1 to -4 annual cycles (histogram); the superimposed curve is a Gaussian fit. The standard deviation is $0.4 \%$

output, we consider the distribution of the root mean square temperature variations within periods with the same calibration factors (typically $\simeq 10$ days); this is given in Fig. 14 cumulatively for the four-year data sets.

Considering the obtained mean value of the root mean square detectors' operating temperature variation: $\simeq 0.04{ }^{\circ} \mathrm{C}$, and the known value of the slope of the light output around its value: $\lesssim-0.2 \% /{ }^{\circ} \mathrm{C}$, the relative light output variation is $\lesssim 10^{-4}$, which would correspond to a modulation amplitude $\lesssim 10^{-4} \mathrm{cpd} / \mathrm{kg} / \mathrm{keV}$ (that is $\lesssim 0.5 \%$ of the observed modulation amplitude).

Thus, there is no significant effect from possible temperature variation in the DAMA/LIBRA installation; on the other hand, in that case some of the specific requirements of the DM annual modulation signature (such as e.g. the 4th and the 5th) would fail, while they are instead satisfied by the DAMA/LIBRA production data. 
Fig. 14 Distribution of the root mean square (r.m.s.) detectors' operating temperature variations calibration factors (typically $\simeq$ 10 days) during the DAMA/LIBRA-1 to -4 annual cycles. The mean value is $0.04^{\circ} \mathrm{C}$ within periods with the same

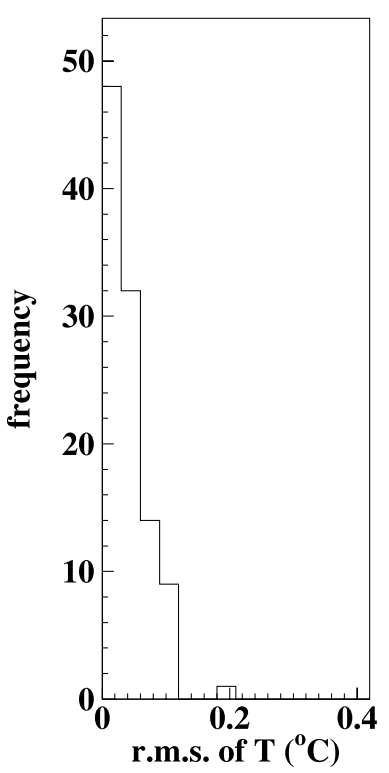

In conclusion, all the arguments given above quantitatively exclude any role of possible effects on the observed rate modulation directly correlated with temperature.

For the sake of completeness, we comment that sizable temperature variations in principle might also induce variations in the electronic noise, in the Radon release from the rocks and in some environmental background; these specific topics will be further addressed in the following.

\subsection{The background}

As quantitatively discussed in previous section, in order to verify the absence of any significant background modulation, the energy distribution measured during the data taking periods in energy regions not of interest for DM detection has been investigated. The presence of background (of whatever nature) modulation is already excluded by the results - given in previous section - on the measured rate integrated above $90 \mathrm{keV}, R_{90}$, as a function of the time; the latter one not only does not show any modulation, but allows one to exclude the presence of a background modulation in the whole energy spectrum at a level some orders of magnitude lower than the annual modulation observed in the single-hit events in the (2-6) keV energy region.

A further relevant support is given by the result of the analysis of the multiple-hits events (see above) which independently proofs that there is no modulation at all in the background event in the same energy region where the single-hit events present an annual modulation satisfying all the requirements of the DM annual modulation signature.

The results given above obviously already account for whatever kind of background including that possibly induced by neutrons, by Radon and by side reactions. In the following we will focus our attention on the latter ones to offer independent cautious analyses to estimate their possible contribution, as done in Refs. $[10,11]$ for the DAMA/NaI data.

\subsection{1 ...more on neutrons}

As regards the thermal neutrons, the neutron capture reactions ${ }^{23} \mathrm{Na}(n, \gamma){ }^{24} \mathrm{Na}$ and ${ }^{23} \mathrm{Na}(n, \gamma){ }^{24 m} \mathrm{Na}$ (cross sections to thermal neutrons equal to 0.10 and 0.43 barn, respectively [114]) have been investigated. The ${ }^{24} \mathrm{Na}$ isotope is a $\beta$-emitter (end point equal to $1.391 \mathrm{MeV}$ ) with two prompt associated $\gamma$ 's (2.754 and $1.369 \mathrm{MeV}$ ), while the ${ }^{24 m} \mathrm{Na}$ isotope decays $100 \%$ of the times in ${ }^{24} \mathrm{Na}$ by internal transition with a $\gamma$ of $0.472 \mathrm{MeV}$. Thus, the possible presence of ${ }^{24} \mathrm{Na}$ isotopes in the $\mathrm{NaI}(\mathrm{Tl})$ crystals gives information about the thermal neutron flux surviving the DAMA/LIBRA shielding and impinging on the DAMA/LIBRA detectors; hence, as reported in Ref. [110], this has been investigated with high sensitivity by looking for triple coincidences induced by a $\beta$ in one detector and by the two $\gamma$ 's in two adjacent ones. An upper limit on the thermal neutron flux surviving the multicomponent DAMA/LIBRA shield has been derived as [110]: $<1.2 \times 10^{-7} \mathrm{~cm}^{-2} \mathrm{~s}^{-1}$ (90\% C.L.). ${ }^{1}$ The corresponding capture rate is: $<0.022$ captures/day $/ \mathrm{kg}$. Assuming cautiously a $10 \%$ modulation (of whatever origin) of the thermal neutrons flux, the corresponding modulation amplitude in the lowest energy region has been calculated by MonteCarlo program to be $<0.8 \times 10^{-6} \mathrm{cpd} / \mathrm{kg} / \mathrm{keV}$ (that is $<0.01 \%$ of the observed modulation amplitude). In addition, a similar contribution cannot anyhow mimic the annual modulation signature since possible modulation of thermal neutron captures would induce e.g. variations in all the energy spectrum, that is it would fail some of the six requirements of the annual modulation signature (such as e.g. the 4th and the 5th).

A similar analysis has also been carried out for the fast neutrons case, as already done for DAMA/NaI [10, 11]. In particular, very safely, the effect of the about $1 \mathrm{~m}$ concrete (made from the Gran Sasso rock material) which almost fully surrounds (outside the barrack) the DAMA/LIBRA passive shield-acting as a further neutron moderator-is not cautiously included here. Thus, from the fast neutron flux measured at the Gran Sasso underground laboratory, $\simeq 10^{-7}$ fast neutrons $\mathrm{cm}^{-2} \mathrm{~s}^{-1}$ [116] the differential counting rate above $2 \mathrm{keV}$ has been estimated by MonteCarlo code to be $\simeq 10^{-3} \mathrm{cpd} / \mathrm{kg} / \mathrm{keV}$. Therefore, assuming cautiously a $10 \%$ modulation (of whatever origin) of the fast neutron flux, the corresponding modulation amplitude is $<10^{-4} \mathrm{cpd} / \mathrm{kg} / \mathrm{keV}$.

\footnotetext{
${ }^{1}$ We remind that the thermal neutron flux has been measured in the LNGS to be $1.08 \times 10^{-6}$ neutrons $\mathrm{cm}^{-2} \mathrm{~s}^{-1}$ [115] and two consistent upper limits on the thermal neutron flux have been obtained with the DAMA/NaI considering the same capture reactions and using different approaches [9, 27].
} 
Fig. 15 Time behaviors of the Radon in the inner part of the barrack (from which-in addition - the detectors are further isolated by other two levels of sealing [110]) during the DAMA/LIBRA-1 to -4 annual cycles, respectively. The measured values are at the level of sensitivity of the used Radon-meter. The same time scale as in Fig. 2 is adopted
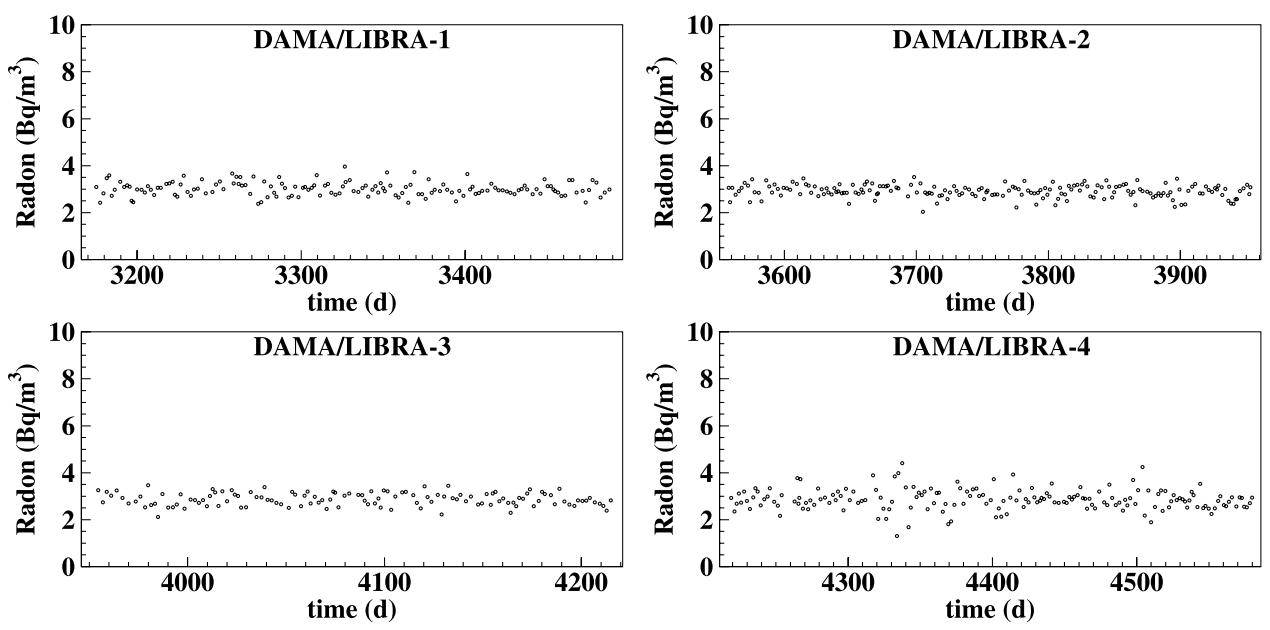

Note that the use of other measurements of fast neutron flux at LNGS $[115,117,118]$ does not change the given conclusions. Moreover, an independent evaluation of the fast neutron flux impinging on the DAMA/LIBRA detectors has been performed by using the DAMA/LIBRA production data through the study of the inelastic reaction ${ }^{23} \mathrm{Na}\left(n, n^{\prime}\right)^{23} \mathrm{Na}^{*}(2076 \mathrm{keV})$, which produces two $\gamma$ 's in coincidence (1636 keV and $440 \mathrm{keV}$ ). An upper limit-limited by the sensitivity of the method-has been found: $<2.2 \times 10^{-7}$ fast neutrons $\mathrm{cm}^{-2} \mathrm{~s}^{-1}$ (90\% C.L.), well compatible with the measured values in the laboratory in Refs. [115-118] and estimated in Ref. [119]. This further excludes any presence of a fast neutron flux in DAMA/LIBRA significantly larger than measured in Refs. [115-118].

It is worth noting that a possible neutron flux modulation as claimed in Ref. [120] at LNGS ( 5\%) and in Ref. [121] at the shallow deep Baradello mine or suggested by phenomenological approaches $[119]^{2}$ cannot quantitatively contribute to the DAMA/LIBRA observed effect, even if the neutron flux at LNGS would be assumed two orders of magnitude larger than measured.

Finally, a possible modulation in the fast neutron flux would induce variation in all the energy spectrum and in the multiple-hits events at low energy, that is some of the six requirements mentioned above would fail.

\subsection{2 ... more on radon}

The DAMA/LIBRA detectors are excluded from the air of the underground laboratory by a 3-level sealing system [110]; in fact, this air contains traces of the radioactive Radon gas $\left({ }^{222} \mathrm{Rn}-\mathrm{T}_{1 / 2}=3.82\right.$ days - and of ${ }^{220} \mathrm{Rn}-$ $\mathrm{T}_{1 / 2}=55 \mathrm{~s}$-isotopes, which belong to the ${ }^{238} \mathrm{U}$ and ${ }^{232} \mathrm{Th}$

\footnotetext{
${ }^{2}$ In particular, it has been suggested that a possible origin of the variability of the neutron flux might be ascribed to possible variations of the water content of the environment.
}

chains, respectively), whose daughters attach themselves to surfaces by various processes. In particular: (i) the walls, the floor and the top of the inner part of the installation are insulated by Supronyl (permeability: $2 \times 10^{-11} \mathrm{~cm}^{2} / \mathrm{s}$ [122]) and a large flux of HP Nitrogen is released in the closed space of this inner part of the barrack housing the set-up. An Oxygen level alarm informs the operator before entering it, when necessary; (ii) the whole passive shield is sealed in a Plexiglas box and maintained continuously in HP Nitrogen atmosphere in slight overpressure with respect to the environment as well as the upper glove box for calibrating the detectors; (iii) the detectors are housed in an inner sealed $\mathrm{Cu}$ box also maintained continuously in HP Nitrogen atmosphere in slight overpressure with respect to the environment; the $\mathrm{Cu}$ box can enter in contact only with the upper glove boxduring calibrations-which is also continuously maintained in HP Nitrogen atmosphere in slightly overpressure with respect to the external environment.

Notwithstanding the above considerations, the Radon in the installation outside the Plexiglas box, containing the passive shield, is continuously monitored; it is at level of sensitivity of the used Radon-meter. The time behaviors for the DAMA/LIBRA-1, $-2,-3$, and -4 annual cycles are shown in Fig. 15. As quantitatively reported in Table 3, no modulation of Radon is present in the environment of the set-up; moreover, the detectors are further isolated by the other two levels of sealing [110].

In Fig. 16 the distributions of the relative variations of the HP Nitrogen flux in the inner $\mathrm{Cu}$ box housing the detectors and of its pressure as measured during the DAMA/LIBRA-1 to -4 annual cycles are shown (the typical flux mean value for each annual cycle is of order of $\simeq 320 \mathrm{l} / \mathrm{h}$ and the typical overpressure mean value is of order of $3.1 \mathrm{mbar}$ ).

We have also investigated possible Radon trace in the HP Nitrogen atmosphere inside the $\mathrm{Cu}$ box, housing the detectors, by searching for the double coincidences of the gammarays (609 and $1120 \mathrm{keV}$ ) from ${ }^{214} \mathrm{Bi}$ Radon daughter, ob- 

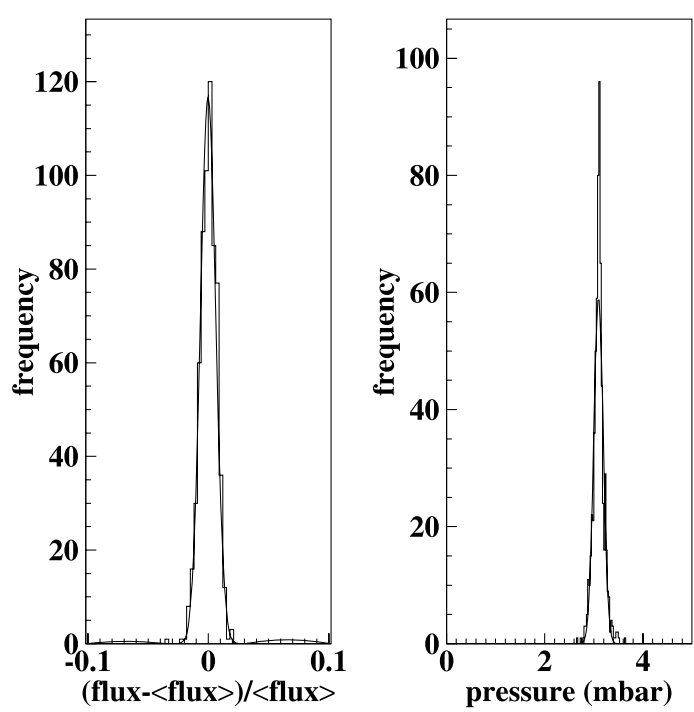

Fig. 16 Distributions of the HP Nitrogen flux in the inner $\mathrm{Cu}$ box housing the detectors and of its pressure as measured during the DAMA/LIBRA-1 to -4 annual cycles (histograms); the superimposed curves are Gaussian fits. For clarity the HP Nitrogen flux has been given in terms of relative variations

taining an upper limit on the possible Radon concentration in the $\mathrm{Cu}$ box HP Nitrogen atmosphere: $<5.8 \times 10^{-2} \mathrm{~Bq} / \mathrm{m}^{3}$ (90\% C.L.); thus, roughly $<2.5 \times 10^{-5} \mathrm{cpd} / \mathrm{kg} / \mathrm{keV}$ can be expected from this source in the lowest energy bins and single-hit events in DAMA/LIBRA as of interest for DM detection. The improvement-with respect to DAMA/NaI—of the limit of the expected contribution to the single-hit events counting rate at low energy is due to the enlarged matrix of detectors and to the better filling of the $\mathrm{Cu}$ box, housing the detectors, e.g. thanks to the new $\mathrm{Cu}$ shaped shield for PMTs and light guides [110].

This shows that even an hypothetical, e.g. 10\%, modulation of possible Radon in the HP Nitrogen atmosphere of the $\mathrm{Cu}$ box, housing the detectors, would correspond to a modulation amplitude $<2.5 \times 10^{-6} \mathrm{cpd} / \mathrm{kg} / \mathrm{keV}$ ( $<0.01 \%$ of the observed modulation amplitude).

Moreover, it is worth noting that, while the possible presence of a sizable quantity of Radon nearby a detector would forbid the investigation of the annual modulation signature (since every Radon variation would induce both the variation in the whole energy distribution and the continuous pollution of the exposed surfaces by the non-volatile daughters), it cannot mimic the DM annual modulation signature in experiments such as the former DAMA/NaI and the present DAMA/LIBRA which record the whole energy distribution; in fact, possible presence of Radon variation can easily be identified in this case and some of the six requirements of the DM annual modulation signature would fail.

In conclusion, no significant effect is possible from the Radon.

\subsection{3 ...more on side processes}

Finally, possible side reactions have also been carefully searched for. The only process which has been found as an hypothetical possibility is the muon flux modulation reported by the MACRO experiment [123]. In fact, MACRO has observed that the muon flux shows a nearly sinusoidal time behavior with one year period and maximum in the summer with amplitude of $\simeq 2 \%$; this muon flux modulation is correlated with the temperature of the Earth's atmosphere. A simple calculation to estimate the modulation amplitude expected from this process in the DAMA/LIBRA set-up can follow the analysis introduced in Refs. $[10,11]$ and recalled in the following. The muon flux $\left(\Phi_{\mu}\right)$ and the yield of neutrons produced by muons measured at the underground Gran Sasso National Laboratory $(Y)$ are: $\Phi_{\mu} \simeq$ 20 muons $\mathrm{m}^{-2} \mathrm{~d}^{-1}[123]$ and $Y \simeq(1-7) \times 10^{-4}$ neutrons per muon per $\mathrm{g} / \mathrm{cm}^{2}[124,125]$, respectively. Thus, the fast neutron rate produced by muons is given by: $R_{n}=\Phi_{\mu} \cdot Y$. $M_{\text {eff }}$, where $M_{\text {eff }}$ is the effective mass where muon interactions can give rise to events detected in the DAMA set-up. Consequently, the annual modulation amplitude in the lowest energy region induced in DAMA/LIBRA by a muon flux modulation as measured by MACRO [123] can be estimated according to: $S_{m}^{(\mu)}=R_{n} \cdot g \cdot \epsilon \cdot f_{\Delta E} \cdot f_{\text {single }} \cdot 2 \% /\left(M_{\text {set-up }}\right.$. $\Delta E$ ), where $g$ is a geometrical factor, $\epsilon$ is the detection efficiency for elastic scattering interactions, $f_{\triangle E}$ is the acceptance of the considered energy window $(E \geq 2 \mathrm{keV}), f_{\text {single }}$ is the single-hit efficiency and $2 \%$ is the MACRO measured effect. Since $M_{\text {set-up }} \simeq 250 \mathrm{~kg}$ and $\Delta E \simeq 4 \mathrm{keV}$, assuming the very cautious values $g \simeq \epsilon \simeq f_{\Delta E} \simeq f_{\text {single }} \simeq 0.5$ and $M_{\text {eff }}=15$ t, one obtains: $S_{m}^{(\mu)}<(0.4-3) \times 10^{-5} \mathrm{cpd} / \mathrm{kg} / \mathrm{keV}$. We stress that-in addition- the latter value has been overestimated of orders of magnitude both because of the extremely cautious values assumed in the calculation and, as mentioned, of the omission of the effect of the $\simeq 1 \mathrm{~m}$ concrete neutron moderation. Finally, we remark that not only the modulation of the muon flux observed by MACRO would give rise in our set-up to a quantitatively negligible effect, but-in addition - some of the six requirements necessary to mimic the annual modulation signature (such as e.g. the 4 th and the 5th) would fail. Therefore, it can be safely ignored.

Just for the sake of completeness, we remind that the contribution of solar neutrinos, whose flux is also expected to be modulated, is many orders of magnitude lower than the modulation amplitude measured by DAMA/LIBRA [28, 29].

\subsection{The noise}

Despite the good noise identification near energy threshold and the stringent noise rejection procedure which is used [110], the role of a possible noise tail in the data after the 
Fig. 17 Time behaviors of the variable

$R_{H}=\sum_{j}\left(R_{H j}-\left\langle R_{H j}\right\rangle\right)$, where $R_{H j}$ is the hardware rate of each detector above single photoelectron threshold (that is including the noise), $j$ identifies the detector and $\left\langle R_{H j}\right\rangle$ is the mean value of $R_{H j}$ in the corresponding annual cycle. The same time scale as in Fig. 2 is adopted
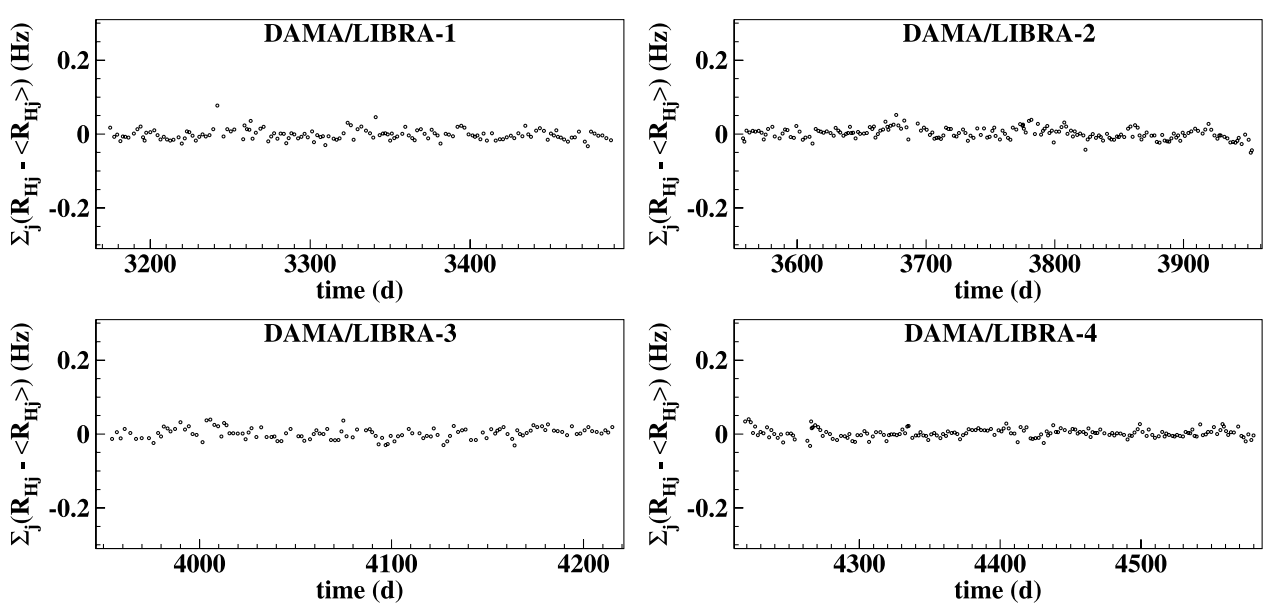

noise rejection procedure has been quantitatively investigated.

In particular, the hardware rate of each detector above a single photoelectron, $R_{H j}$ ( $j$ identifies the detector), has been considered. Indeed, this hardware rate is significantly determined by the noise.

For the proposed purpose the variable: $R_{H}=\sum_{j}\left(R_{H j}-\right.$ $\left\langle R_{H j}\right\rangle$ ), can be built; in the present case $\left\langle R_{H j}\right\rangle \lesssim 0.2 \mathrm{~Hz}$. The time behaviors of $R_{H}$ during the DAMA/LIBRA-1 to -4 annual cycles are shown in Fig. 17.

As can be seen in Fig. 18, the cumulative distribution of $R_{H}$ for the DAMA/LIBRA-1 to -4 annual cycles shows a Gaussian behavior with $\sigma=0.3 \%$, value well in agreement with the one expected on the basis of simple statistical arguments.

Moreover, by fitting the time behavior of $R_{H}$ in the four data taking periods-including a modulation term as that for DM particles - a modulation amplitude compatible with zero: $(0.03 \pm 0.09) \times 10^{-2} \mathrm{~Hz}$, is obtained. From this value the upper limit at $90 \%$ C.L. on the modulation amplitude can be derived: $<1.8 \times 10^{-3} \mathrm{~Hz}$. Since the typical noise contribution to the hardware rate of each detector is $\simeq 0.10 \mathrm{~Hz}$, the upper limit on the noise relative modulation amplitude is given by: $\frac{1.8 \times 10^{-3} \mathrm{~Hz}}{2.5 \mathrm{~Hz}} \simeq 7.2 \times 10^{-4}$ (90\% C.L.). Therefore, even in the worst hypothetical case of a $10 \%$ contamination of the residual noise-after rejection-in the counting rate, the noise contribution to the modulation amplitude in the lowest energy bins would be $<7.2 \times 10^{-5}$ of the total counting rate. This means that an hypothetical noise modulation could account at maximum for absolute amplitudes less than $10^{-4} \mathrm{cpd} / \mathrm{kg} / \mathrm{keV}$.

In conclusion, there is no evidence for any role of an hypothetical tail of residual noise after rejection.

\subsection{The calibration factor}

The performed calibrations have been discussed in Ref. [110]; in particular, in long term running conditions
Fig. 18 Distribution of $R_{H}$ during the DAMA/LIBRA-1 to -4 annual cycles (histogram); the superimposed curve is a Gaussian fit. See text

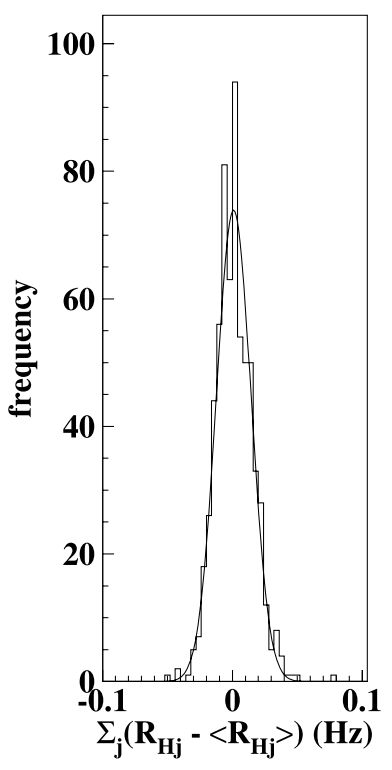

periodical calibrations are performed every $\simeq 10$ days with ${ }^{241}$ Am source.

Although it is highly unlikely that a variation of the calibration factor (proportionality factor between the area of the recorded pulse and the energy), tdcal, could play any role, a quantitative investigation on that point has been carried out.

For this purpose, we define the percentage variation of each energy scale factor ( $t d c a l$ ) with respect to the value measured in the previous calibration: $\epsilon_{t d c a l}=\frac{t d c a l_{k}-t_{d c a l}-1}{t_{d c a l} l_{k-1}}$ (here $t_{\text {cal }}$ is the value of the calibration factor in the $k$ th calibration). The distribution of $\epsilon_{t d c a l}$ for all the detectors during the DAMA/LIBRA-1 to -4 annual cycles is given in Fig. 19(left). This distribution shows a Gaussian behavior with $\sigma \simeq 0.5 \%$. Since the results of the routine calibrations are properly taken into account in the data analysis, such a result allows us to conclude that the energy calibration factor for each detector is known with an uncertainty $\ll 1 \%$ during the data taking periods. 

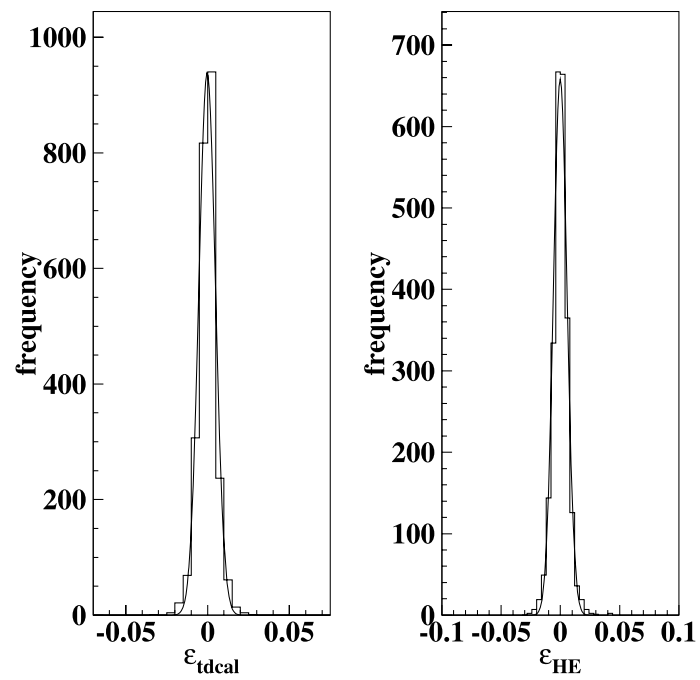

Fig. $19 L e f t$ : Distribution of the percentage variations $\left(\epsilon_{t d c a l}\right)$ of each energy scale factor ( $t d c a l)$ with respect to the value measured in the previous calibration for the DAMA/LIBRA-1 to -4 annual cycles (histogram); the superimposed curve is a Gaussian fit. The standard deviation is $0.5 \%$. Right: Distribution of the percentage variations $\left(\epsilon_{H E}\right)$ of the high energy scale factor with respect to the mean values for the DAMA/LIBRA-1 to -4 annual cycles (histogram); the superimposed curve is a Gaussian fit. The standard deviation is $0.6 \%$

Moreover, the distribution of the percentage variations $\left(\epsilon_{H E}\right)$ of the high energy scale factor with respect to the mean values for all the detectors and for the DAMA/LIBRA1 to -4 annual cycles is reported in Fig. 19(right). Also this distribution shows a Gaussian behavior with $\sigma \simeq 0.6 \%$.

As discussed also in Refs. [10, 11], the possible variation of the calibration factor for each detector during the data taking would give rise to an additional energy spread $\left(\sigma_{c a l}\right)$ besides the detector energy resolution $\left(\sigma_{\text {res }}\right)$. The total energy spread can be, therefore, written as: $\sigma=\sqrt{\sigma_{\text {res }}^{2}+\sigma_{\text {cal }}^{2}} \simeq$ $\sigma_{\text {res }} \cdot\left[1+\frac{1}{2} \cdot\left(\frac{\sigma_{\text {cal }}}{\sigma_{\text {res }}}\right)^{2}\right]$; clearly the contribution due to the calibration factor variation is negligible since $\frac{1}{2} \cdot\left(\frac{\sigma_{c a l} / E}{\sigma_{\text {res }} / E}\right)^{2} \lesssim$ $7.5 \times 10^{-4} \frac{E}{20 \mathrm{keV}}$ (where the adimensional ratio $\frac{E}{20 \mathrm{keV}}$ accounts for the energy dependence of this limit value). This order of magnitude is confirmed by a MonteCarlo calculation, which credits—as already reported in Refs. [10, 11]—a maximum value of the effect of similar variations of $t d c a l$ on the modulation amplitude equal to $1-2 \times 10^{-4} \mathrm{cpd} / \mathrm{kg} / \mathrm{keV}$. Thus, also the unlikely idea that the calibration factor could play a role can be safely ruled out.

\subsection{The efficiencies}

The behavior of the overall efficiencies during the whole data taking periods has even been investigated. Their possible time variation depends essentially on the stability of the efficiencies related to the adopted acceptance windows; they are regularly measured by dedicated calibrations [110].
Fig. 20 Distribution of the percentage variations of the overall efficiency values with the respect to their mean values during the DAMA/LIBRA-1 to -4 annual cycles (histogram); the superimposed curve is a Gaussian fit. See text

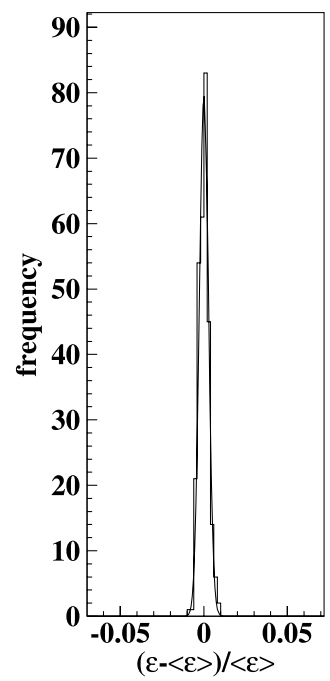

In particular, Fig. 20 shows the percentage variations of the efficiency values in the (2-8) $\mathrm{keV}$ energy interval considering $2 \mathrm{keV}$ bins. They show a Gaussian distribution with $\sigma=0.3 \%$ for DAMA/LIBRA- 1 to -4 , cumulatively. Moreover, we have verified that the time behavior of these percentage variations does not show any modulation with period and phase expected for a possible DM signal. In Table 4 the modulation amplitudes of the efficiencies in each energy bin between 2 and $10 \mathrm{keV}$ are reported, showing that they are all consistent with zero. In particular, modulation amplitudes-considering the four DAMA/LIBRA annual cycles all together-equal to $(0.1 \pm 0.4) \times 10^{-3}$ and $-(0.4 \pm 0.4) \times 10^{-3}$ are found for the (2-4) $\mathrm{keV}$ and (4-6) keV energy bins, respectively; both consistent with zero. Thus, also the unlikely idea of a possible role played by the efficiency values is ruled out.

\subsection{Conclusions on possible systematics effects}

No modulation has been found in any possible source of systematics or side reactions; thus, upper limits (90\% C.L.) on the possible contributions to the DAMA/LIBRA measured modulation amplitude are summarized in Table 5. In particular, they cannot account for the measured modulation both because quantitatively not relevant and unable to mimic the observed effect.

\section{Conclusions}

The model independent results achieved by the second generation DAMA/LIBRA set-up in operation at the Gran Sasso National Laboratory confirms evidence of Dark Matter particles in the galactic halo with high confidence level; a cumulative C.L. of $8.2 \sigma$ is reached when considering the data of the former DAMA/NaI experiment and the present ones 
Table 4 Modulation amplitudes obtained by fitting the time behavior of the efficiencies including a cosine modulation with phase and period as for DM particles for the DAMA/LIBRA-1 to -4 annual cycles

\begin{tabular}{llclr}
\hline Energy & Amplitudes $\left(\times 10^{-3}\right)$ & & \\
\cline { 2 - 5 } & DAMA/LIBRA-1 & DAMA/LIBRA-2 & DAMA/LIBRA-3 & $-(0.4 \pm 1.0)$ \\
\hline $2-4 \mathrm{keV}$ & $(0.3 \pm 0.6)$ & $(0.1 \pm 0.6)$ & $-(0.4 \pm 1.1)$ & $-(0.7 \pm 1.0)$ \\
$4-6 \mathrm{keV}$ & $(0.0 \pm 0.6)$ & $-(0.7 \pm 0.6)$ & $-(0.3 \pm 1.0)$ & $-(1.0 \pm 0.8)$ \\
$6-8 \mathrm{keV}$ & $-(0.3 \pm 0.6)$ & $-(1.0 \pm 0.7)$ & $-(0.2 \pm 0.6)$ & $(0.7 \pm 0.6)$ \\
$8-10 \mathrm{keV}$ & $-(0.5 \pm 0.5)$ & $-(0.5 \pm 0.5)$ & & \\
\hline
\end{tabular}

Table 5 Summary of the results obtained by investigating possible sources of systematics or of side reactions in the data of the DAMA/LIBRA-1 to -4 annual cycles. None able to give a modula- tion amplitude different from zero has been found; thus cautious upper limits ( $90 \%$ C.L.) on the possible contributions to the measured modulation amplitude have been calculated and are shown here

\begin{tabular}{lll}
\hline Source & Main comment (see also Ref. [110]) & Cautious upper limit (90\%C.L.) \\
\hline Radon & Sealed Cu Box in & $<2.5 \times 10^{-6} \mathrm{cpd} / \mathrm{kg} / \mathrm{keV}$ \\
& HP Nitrogen atmosphere, & \\
& 3-level of sealing & $<10^{-4} \mathrm{cpd} / \mathrm{kg} / \mathrm{keV}$ \\
Temperature & Air conditioning & \\
& + huge heat capacity & $<10^{-4} \mathrm{cpd} / \mathrm{kg} / \mathrm{keV}$ \\
Noise & Efficient rejection & $<1-2 \times 10^{-4} \mathrm{cpd} / \mathrm{kg} / \mathrm{keV}$ \\
Energy scale & Routine & $<10^{-4} \mathrm{cpd} / \mathrm{kg} / \mathrm{keV}$ \\
& + intrinsic calibrations & $<10^{-4} \mathrm{cpd} / \mathrm{kg} / \mathrm{keV}$ \\
Efficiencies & Regularly measured & \\
Background & No modulation above $6 \mathrm{keV} ;$ & \\
& no modulation in the $(2-6) \mathrm{keV}$ & $<3 \times 10^{-5} \mathrm{cpd} / \mathrm{kg} / \mathrm{keV}$ \\
& multiple-hit events; &
\end{tabular}

of DAMA/LIBRA all together. In particular, deep quantitative analyses exclude any effect either from systematics or from side processes (temperature, noise, hardware or software procedures, background of whatever nature including also Radon, neutrons and cosmic rays). We note that no experiment exists whose result can be directly compared with those presented here.

The wide sensitivity of the $\mathrm{NaI}(\mathrm{Tl})$ target-detector material to many of the possible DM candidates and of the possible astrophysical, nuclear and particle Physics scenarios, the reached intrinsic radiopurity, the used approach, the specific performances and operating conditions, the large collected exposures of the former DAMA/NaI and of the present DAMA/LIBRA set-ups, have offered an unique possibility of an effective model independent investigation.

Model dependent considerations will be presented in later publication specifically devoted to this aspect. Just few arguments for some illustrative purposes are given in Appendix.

The collection of a larger exposure with DAMA/LIBRA (and with the possible DAMA/1 ton, which is at R\&D stage) will also allow the improvement of corollary information which can be derived on the nature of the candidate particle(s) and on the various related astrophysical, nuclear and particle Physics scenarios, and the investigation with very high sensitivity of the other DM features and second order effects as well as of several rare processes other than DM.

\section{Appendix}

As in the past (see e.g. [11-18]), corollary investigations can also be pursued-on the basis of the cumulative $8.2 \sigma$ C.L. model-independent result by DAMA/NaI 

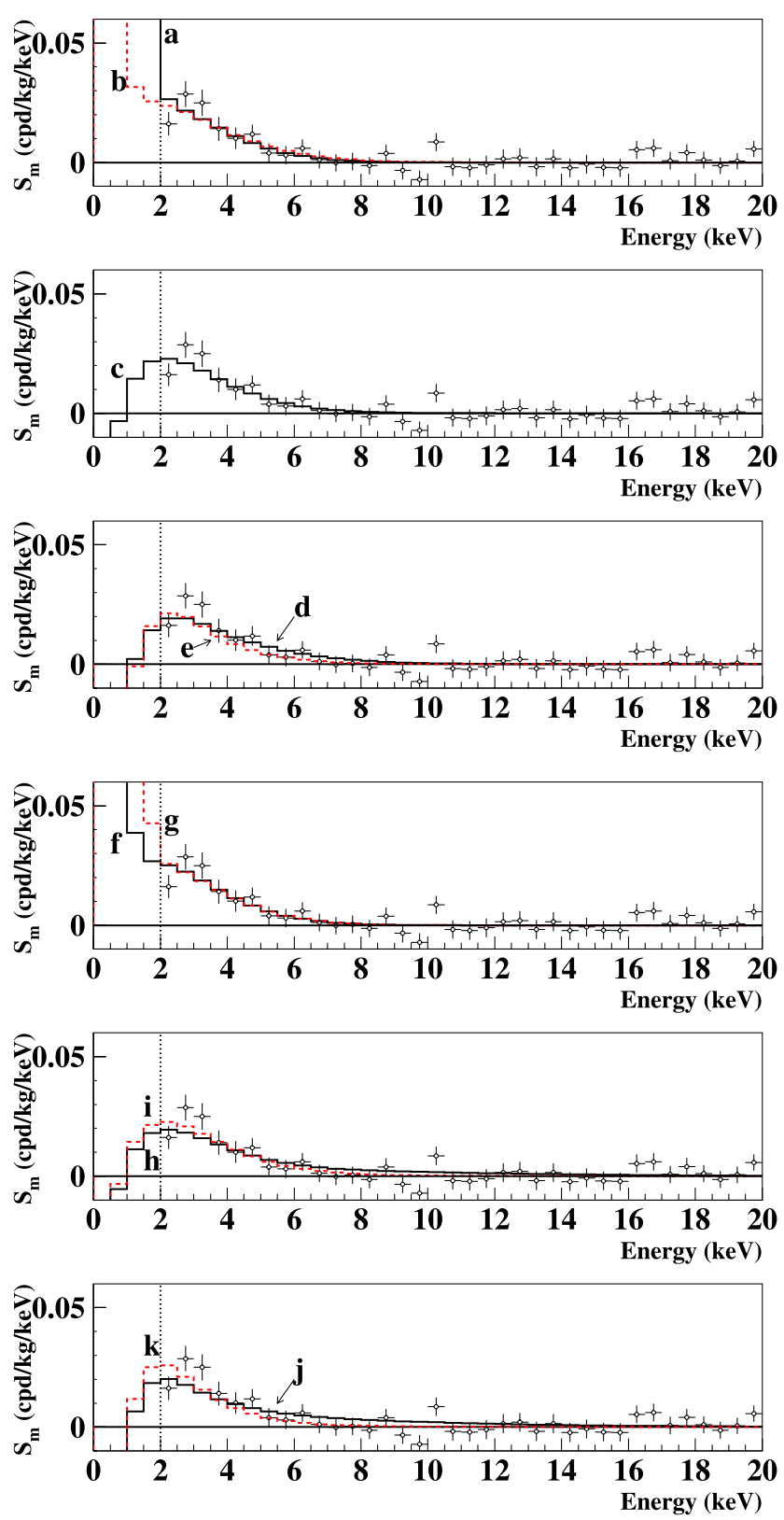

Fig. 21 Few examples of expected behaviors for few of the many possible scenarios (see Table 6), superimposed to the measured $S_{m, k}$ values of Fig. 9. The shown behaviors have not been obtained by the maximum likelihood method (see in our quoted literature) and are shown just for illustrative purpose; they all give practically about the same C.L. As mentioned in the text, the full treatment of the data by maximum likelihood method to update the volumes/regions allowed at given C.L. by the cumulative DAMA/NaI and DAMA/LIBRA data for the considered scenarios will be presented elsewhere following the full analysis method of Refs. [11-18]

and DAMA/LIBRA - on the nature of the DM candidate particle and on related astrophysical, nuclear and particle Physics scenarios. As widely discussed elsewhere, these investigations are instead model-dependent and—considering the large uncertainties which exist on the astrophysical, nu-
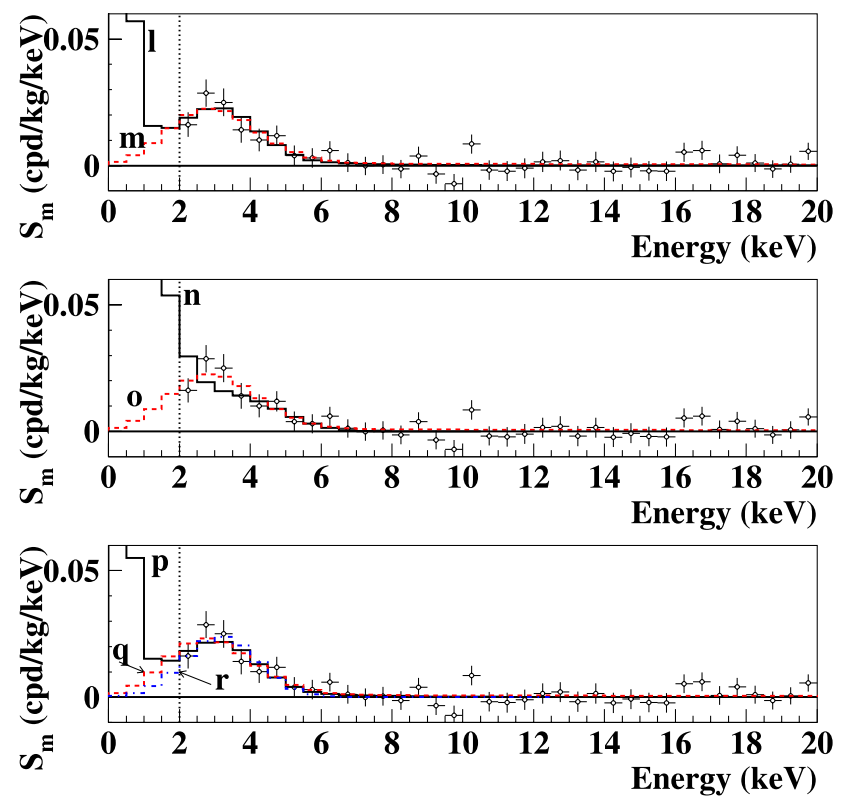

Fig. 21 (Continued)

clear and particle physics assumptions and on the theoretical and experimental parameters needed in the calculationshave no general meaning (as it is also the case of exclusion plots and of the DM particle parameters evaluated in indirect detection experiments).

Complete model dependent analyses, to update the allowed regions in various scenarios and to enlarge the investigations to other ones, will be presented elsewhere. Here, we just remind that many astrophysical, nuclear and particle physics scenarios and many DM particle candidates exist. Just to offer some naive feeling on the complexity of the argument, we show in Fig. 21 the experimental $S_{m}$ values of Fig. 9 with superimposed the expected behaviors for some DM candidates in few of the many possible scenarios and parameters values (see Table 6). In fact, despite the behavior of the $S_{m}$ values can be effective a posteriori for template purpose, very accurate results on corollary model dependent quests at given C.L. should be evaluated by applying the maximum likelihood analysis in time and energy of all the events (as described elsewhere), which offers efficient and complete data analyses accounting for all the experimental information carried out by the data and, when of interest, for priors.

It is worth noting that an increase of the exposure and a possible lowering of the used $2 \mathrm{keV}$ threshold will improve the discrimination capability among different astrophysical, nuclear and particle Physics scenarios.

Let us, finally, note that results obtained with different target materials and/or different approaches cannot intrinsically be directly compared among them even when considering the same kind of candidate and of coupling, although 
Table 6 Scenarios and parameters values used in Fig. 21; they have been chosen among the many possible ones [11-18]. In the fourth column the considered Set—as in Ref. [11]—of nuclear form factors and/or of nuclear quenching factors is reported. Here: (i) $\sigma_{S I}$ is the spin independent point-like cross section; (ii) $\sigma_{S D}$ is the spin dependent point-like cross section; (iii) $\theta$ is an angle defined in the $[0, \pi)$ interval, whose tangent is the ratio between the WIMP-neutron and the WIMPproton effective SD coupling strengths, respectively [11]; (iv) $m_{H}$ is the mass of the LDM particle; (v) $\Delta$ is the mass splitting [18]; (vi) $g_{a e e}$ is the bosonic axion-like particle coupling to electrons. For the cross sections of the LDM particle see Ref. [18] and for the channeling effect see Ref. [16]

DM particle elastic scattering on nuclei, spin-independent (SI) and spin-dependent (SD) couplings,

local velocity $=170 \mathrm{~km} / \mathrm{s}$ and nuclear cross section scaling laws as in [11]

\begin{tabular}{|c|c|c|c|c|c|c|c|c|}
\hline $\begin{array}{l}\text { Curve } \\
\text { label }\end{array}$ & $\begin{array}{l}\text { Halo model } \\
\text { (see Refs. }[11,126])\end{array}$ & $\begin{array}{l}\text { Local density } \\
\left(\mathrm{GeV} / \mathrm{cm}^{3}\right)\end{array}$ & $\begin{array}{l}\text { Set as } \\
\text { in [11] }\end{array}$ & $\begin{array}{l}\text { DM particle } \\
\text { mass }\end{array}$ & $\begin{array}{l}\xi \sigma_{S I} \\
(\mathrm{pb})\end{array}$ & $\begin{array}{l}\xi \sigma_{S D} \\
(\mathrm{pb})\end{array}$ & $\begin{array}{l}\theta \\
(\mathrm{rad})\end{array}$ & $\begin{array}{l}\text { Channeling } \\
{[16]}\end{array}$ \\
\hline$a$ & A5 (NFW) & 0.2 & A & $15 \mathrm{GeV}$ & $3.1 \times 10^{-4}$ & 0 & - & no \\
\hline$b$ & A5 (NFW) & 0.2 & A & $15 \mathrm{GeV}$ & $1.3 \times 10^{-5}$ & 0 & - & yes \\
\hline$c$ & A5 (NFW) & 0.2 & B & $60 \mathrm{GeV}$ & $5.5 \times 10^{-6}$ & 0 & - & no \\
\hline$d$ & $\begin{array}{l}\text { B3 (Evans } \\
\text { power law) }\end{array}$ & 0.17 & B & $100 \mathrm{GeV}$ & $6.5 \times 10^{-6}$ & 0 & - & no \\
\hline$e$ & $\begin{array}{l}\text { B3 (Evans } \\
\text { power law) }\end{array}$ & 0.17 & A & $120 \mathrm{GeV}$ & $1.3 \times 10^{-5}$ & 0 & - & no \\
\hline$f$ & A5 (NFW) & 0.2 & A & $15 \mathrm{GeV}$ & $10^{-7}$ & 2.6 & 2.435 & no \\
\hline$g$ & A5 (NFW) & 0.2 & A & $15 \mathrm{GeV}$ & $1.4 \times 10^{-4}$ & 1.4 & 2.435 & no \\
\hline$h$ & A5 (NFW) & 0.2 & B & $60 \mathrm{GeV}$ & $10^{-7}$ & 1.4 & 2.435 & no \\
\hline$i$ & A5 (NFW) & 0.2 & B & $60 \mathrm{GeV}$ & $8.7 \times 10^{-6}$ & $8.7 \times 10^{-2}$ & 2.435 & no \\
\hline$j$ & $\begin{array}{l}\text { B3 (Evans } \\
\text { power law) }\end{array}$ & 0.17 & A & $100 \mathrm{GeV}$ & $10^{-7}$ & 1.7 & 2.435 & no \\
\hline$k$ & $\begin{array}{l}\text { B3 (Evans } \\
\text { power law) }\end{array}$ & 0.17 & A & $100 \mathrm{GeV}$ & $1.1 \times 10^{-5}$ & 0.11 & 2.435 & no \\
\hline
\end{tabular}

Light Dark Matter (LDM) inelastic scattering and bosonic axion-like interaction as in [13, 18],

A5 (NFW) halo model as in $[11,126]$, local density $=0.17 \mathrm{GeV} / \mathrm{cm}^{3}$, local velocity $=170 \mathrm{~km} / \mathrm{s}$

\begin{tabular}{|c|c|c|c|c|c|c|c|}
\hline $\begin{array}{l}\text { Curve } \\
\text { label }\end{array}$ & DM particle & Interaction & $\begin{array}{l}\text { Set as } \\
\text { in [11] }\end{array}$ & $m_{H}$ & $\Delta$ & $\begin{array}{l}\text { Cross } \\
\text { section }(\mathrm{pb})\end{array}$ & $\begin{array}{l}\text { Channeling } \\
{[16]}\end{array}$ \\
\hline$l$ & LDM & $\begin{array}{l}\text { coherent } \\
\text { on nuclei }\end{array}$ & A & $30 \mathrm{MeV}$ & $18 \mathrm{MeV}$ & $\xi \sigma_{m}^{c o h}=1.8 \times 10^{-6}$ & yes \\
\hline$m$ & LDM & $\begin{array}{l}\text { coherent } \\
\text { on nuclei }\end{array}$ & A & $100 \mathrm{MeV}$ & $55 \mathrm{MeV}$ & $\xi \sigma_{m}^{c o h}=2.8 \times 10^{-6}$ & yes \\
\hline$n$ & LDM & $\begin{array}{l}\text { incoherent } \\
\text { on nuclei }\end{array}$ & A & $30 \mathrm{MeV}$ & $3 \mathrm{MeV}$ & $\xi \sigma_{m}^{i n c}=2.2 \times 10^{-2}$ & yes \\
\hline$o$ & LDM & $\begin{array}{l}\text { incoherent } \\
\text { on nuclei }\end{array}$ & A & $100 \mathrm{MeV}$ & $55 \mathrm{MeV}$ & $\xi \sigma_{m}^{i n c}=4.6 \times 10^{-2}$ & yes \\
\hline$p$ & LDM & $\begin{array}{l}\text { coherent } \\
\text { on nuclei }\end{array}$ & A & $28 \mathrm{MeV}$ & $28 \mathrm{MeV}$ & $\xi \sigma_{m}^{c o h}=1.6 \times 10^{-6}$ & yes \\
\hline$q$ & LDM & $\begin{array}{l}\text { incoherent } \\
\text { on nuclei }\end{array}$ & A & $88 \mathrm{MeV}$ & $88 \mathrm{MeV}$ & $\xi \sigma_{m}^{i n c}=4.1 \times 10^{-2}$ & yes \\
\hline$r$ & LDM & on electrons & - & $60 \mathrm{keV}$ & $60 \mathrm{keV}$ & $\xi \sigma_{m}^{e}=0.3 \times 10^{-6}$ & - \\
\hline$r$ & $\begin{array}{l}\text { pseudoscalar } \\
\text { axion-like }\end{array}$ & see Ref. [13] & - & Mass $=3.2 \mathrm{keV}$ & & $g_{a e e}=3.9 \times 10^{-11}$ & - \\
\hline
\end{tabular}


apparently all the presentations generally refer to cross section on nucleon.

For completeness, we also further note that no experiment exists whose result can be directly compared in a model independent way with the ones by DAMA/NaI and DAMA/LIBRA. Thus claims for contradictions are arbitrary, in fact, e.g.: (1) the others are insensitive to the annual modulation signature; (2) the others use different target materials; (3) the plots they show and those they attribute to others are built at a "single cooking" without accounting at all for the existing experimental, theoretical and phenomenological uncertainties and for the existing alternative choices; (4) DAMA/NaI and DAMA/LIBRA have a favored sensitivity with respect to others in several scenarios; moreover, scenarios exist (see literature) to which the others are not only disfavored with respect to the DAMA experiments, but even blind. Furthermore, additional realistic limitations in those claimed model dependent sensitivities (just for "nuclear recoils" and a single assumed scenario and parameters set) can arise also from some experimental aspects; some arguments have been addressed e.g. in Refs. $[11-18,127]$ and in some other literature. On the other hand, whenever there might be in future some correct claim for exclusion in one or more particular astrophysical, nuclear and particle physics model framework(s) and assumed parameters set (but correctly accounting both for experimental and theoretical uncertainties and for the implications of the DAMA model independent results in the considered scenario and assumptions), there will be still many other scenarios, parameters sets and DM candidates which can explain the DAMA/NaI and DAMA/LIBRA model independent results to which other target materials and approaches are disfavored or even blind; thus, this never will exclude the model independent results of the DAMA experiments. In addition, whenever an experiment using the same identical target material and methodological approach would be available, as usual in whatever field of Physics a serious comparison would require-in every case-e.g. a deep investigation of the radiopurity of all the part of the different set-ups, of their specific performances in all the aspects, of the detailed procedures used by each one, etc.

\section{References}

1. R. Bernabei et al., Phys. Lett. B 389, 757 (1996)

2. R. Bernabei et al., Phys. Lett. B 424, 195 (1998)

3. R. Bernabei et al., Phys. Lett. B 450, 448 (1999)

4. P. Belli et al., Phys. Rev. D 61, 023512 (2000)

5. R. Bernabei et al., Phys. Lett. B 480, 23 (2000)

6. R. Bernabei et al., Phys. Lett. B 509, 197 (2001)

7. R. Bernabei et al., Eur. Phys. J. C 23, 61 (2002)

8. P. Belli et al., Phys. Rev. D 66, 043503 (2002)

9. R. Bernabei et al., Nuovo Cimento A 112, 545 (1999)

10. R. Bernabei et al., Eur. Phys. J. C 18, 283 (2000)
11. R. Bernabei et al., Riv. Nuovo Cimento 26(1), 1-73 (2003)

12. R. Bernabei et al., Int. J. Mod. Phys. D 13, 2127 (2004)

13. R. Bernabei et al., Int. J. Mod. Phys. A 21, 1445 (2006)

14. R. Bernabei et al., Eur. Phys. J. C 47, 263 (2006)

15. R. Bernabei et al., Int. J. Mod. Phys. A 22, 3155 (2007)

16. R. Bernabei et al., Eur. Phys. J. C 53, 205 (2008)

17. R. Bernabei et al., Phys. Rev. D 77, 023506 (2008)

18. R. Bernabei et al., preprint ROM2F/2008/02. arXiv:0802.4336 [astro-ph], in publication on Mod. Phys. Lett. A

19. R. Bernabei et al., Phys. Lett. B 408, 439 (1997)

20. P. Belli et al., Phys. Lett. B 460, 236 (1999)

21. R. Bernabei et al., Phys. Rev. Lett. 83, 4918 (1999)

22. P. Belli et al., Phys. Rev. C 60, 065501 (1999)

23. R. Bernabei et al., Nuovo Cimento A 112, 1541 (1999)

24. R. Bernabei et al., Phys. Lett. B 515, 6 (2001)

25. F. Cappella et al., Eur. Phys. J. Direct C 14, 1 (2002)

26. R. Bernabei et al., Eur. Phys. J. A 23, 7 (2005)

27. R. Bernabei et al., Eur. Phys. J. A 24, 51 (2005)

28. R. Bernabei et al., Astropart. Phys. 4, 45 (1995)

29. R. Bernabei, in The Identification of Dark Matter (World Scientific, Singapore, 1997), p. 574

30. P. Belli et al., Nuovo Cimento A 103, 767 (1990)

31. P. Belli et al., Astropart. Phys. 5, 217 (1996)

32. P. Belli et al., Nuovo Cimento C 19, 537 (1996)

33. P. Belli et al., Phys. Lett. B 387, 222 (1996); Phys. Lett. B 389, 783 (1996) (Erratum)

34. R. Bernabei et al., Phys. Lett. B 436, 379 (1998)

35. P. Belli et al., Phys. Lett. B 465, 315 (1999)

36. P. Belli et al., Phys. Rev. D 61, 117301 (2000)

37. R. Bernabei et al., New J. Phys. 2, 15 (2000) 1

38. R. Bernabei et al., Phys. Lett. B 493, 12 (2000)

39. R. Bernabei et al., Nucl. Instrum. Methods A 482, 728 (2002)

40. R. Bernabei et al., Eur. Phys. J. Direct C 11, 1 (2001)

41. R. Bernabei et al., in Beyond the Desert 2003 (Springer, Berlin, 2003), p. 365

42. R. Bernabei et al., Eur. Phys. J. A 27(s01), 35 (2006)

43. R. Bernabei et al., Phys. Lett. B 527, 182 (2002)

44. R. Bernabei et al., Phys. Lett. B 546, 23 (2002)

45. R. Bernabei et al., Nucl. Instrum. Methods A 555, 270 (2005)

46. R. Bernabei et al., Ukr. J. Phys. 51, 1037 (2006)

47. P. Belli et al., Nucl. Phys. A 789, 15 (2007)

48. P. Belli et al., Phys. Rev. C 76, 064603 (2007)

49. R. Bernabei et al., Nuovo Cimento A 110, 189 (1997)

50. R. Bernabei et al., Astropart. Phys. 7, 73 (1997)

51. P. Belli et al., Nucl. Phys. B 563, 97 (1999)

52. P. Belli et al., Astropart. Phys. 10, 115 (1999)

53. R. Bernabei et al., Nucl. Phys. A 705, 29 (2002)

54. P. Belli et al., Nucl. Instrum. Methods A 498, 352 (2003)

55. R. Cerulli et al., Nucl. Instum. Methods A 525, 535 (2004)

56. P. Belli et al., Phys. Lett. B 658, 193 (2008)

57. K.A. Drukier et al., Phys. Rev. D 33, 3495 (1986)

58. K. Freese et al., Phys. Rev. D 37, 3388 (1988)

59. P. Belli, R. Bernabei, C. Bacci, A. Incicchitti, R. Marcovaldi, D. Prosperi, DAMA proposal to INFN Scientific Committee II, 24 April 1990

60. M.W. Goodman, E. Witten, Phys. Rev. D 31, 3059 (1985)

61. W.H. Press, D.N. Spergel, Astrophys. J. 296, 679 (1985)

62. R.L. Gilliland et al., Astrophys. J. 306, 703 (1986)

63. K. Griest, Phys. Rev. D 38, 2357 (1988)

64. K. Griest, Phys. Rev. Lett. 61, 666 (1988)

65. R. Barbieri, M. Frigeni, G.F. Giudice, Nucl. Phys. B 313, 725 (1989)

66. G. Gelmini, P. Gondolo, E. Roulet, Nucl. Phys. B 351, 623 (1991)

67. A. Bottino, V. de Alfaro, N. Fornengo, G. Mignola, S. Scopel, Astropart. Phys. 2, 77 (1994) 
68. D. Smith, N. Weiner, Phys. Rev. D 64, 043502 (2001)

69. D. Tucker-Smith, N. Weiner, Phys. Rev. D 72, 063509 (2005)

70. A. Bottino et al., Phys. Lett. B 402, 113 (1997)

71. A. Bottino et al., Phys. Lett. B 423, 109 (1998)

72. A. Bottino et al., Phys. Rev. D 59, 095004 (1999)

73. A. Bottino et al., Phys. Rev. D 59, 095003 (1999)

74. A. Bottino et al., Astropart. Phys. 10, 203 (1999)

75. A. Bottino et al., Astropart. Phys. 13, 215 (2000)

76. A. Bottino et al., Phys. Rev. D 62, 056006 (2000)

77. A. Bottino et al., Nucl. Phys. B 608, 461 (2001)

78. A. Bottino, F. Donato, N. Fornengo, S. Scopel, Phys. Rev. D 63, 125003 (2001)

79. A. Bottino et al., Phys. Rev. D 67, 063519 (2003)

80. A. Bottino et al., Phys. Rev. D 68, 043506 (2003)

81. A. Bottino et al., Phys. Rev. D 69, 037302 (2004)

82. T. Asaka et al., Phys. Rev. D 58, 023507 (1998)

83. T. Asaka et al., Phys. Rev. D 58, 083509 (1998)

84. R. Volkas, Prog. Part. Nucl. Phys. 48, 161 (2002)

85. R. Foot, hep-ph/0308254

86. K. Belotsky, D. Fargion, M. Khlopov, R.V. Konoplich, hep-ph/0411093

87. D. Hooper, L.T. Wang, Phys. Rev. D 70, 063506 (2004)

88. S. Mitra, Phys. Rev. D 71, 121302 (2005) (R)

89. C. Picciotto, M. Pospelov, Phys. Lett. B 605, 15 (2005)

90. M. Kawasaki, T. Yanagida, Phys. Lett. B 624, 162 (2005)

91. D.V. Ahluwalia-Khalilova, D. Grumiller, Phys. Rev. D 72, 067701 (2005)

92. D.V. Ahluwalia-Khalilova, D. Grumiller, J. Cosmol. Astropart. Phys. 07, 012 (2005)

93. J. Knodlseder et al., Astron. Astrophys. 441, 513 (2005)

94. J.M. Frére et al., arXiv:hep-ph/0610240

95. Y. Ascasibar, P. Jean, C. Boehm, J. Knoedlseder, Mon. Not. R. Astron. Soc. 368, 1695 (2006)

96. E.M. Drobyshevski et al., Astron. Astrophys. Trans. 26(4), 289 (2007)
97. E.M. Drobyshevski et al., arXiv:0704.0982

98. E.M. Drobyshevski et al., arXiv:0706.3095

99. D. Hooper et al., arXiv:0704.2558 [astro-ph]

100. C. Jacoby, S. Nussinov, J. High Energy Phys. 05, 017 (2007)

101. D.P. Finkbeiner, N. Weiner, Phys. Rev. D 76, 083519 (2007)

102. M. Pospelov, A. Ritz, Phys. Lett. B 651, 208 (2007)

103. P. Fayet, Phys. Rev. D 75, 115017 (2007)

104. A. Kusenko, AIP Conf. Proc. 917, 58 (2007)

105. A. Palazzo et al., Phys. Rev. D 76, 103511 (2007)

106. M. Shaposhnikov, Nucl. Phys. B 763, 49 (2007)

107. M. Lemoine et al., Phys. Lett. B 645, 222 (2007)

108. C. Arina, N. Fornengo, arXiv:0709.4477

109. A. Bottino et al., Phys. Rev. D 77, 015002 (2008)

110. R. Bernabei et al., Nucl. Instrum. Methods A 592, 297 (2008)

111. W.H. Press, G.B. Rybicki, Astrophys. J. 338, 277 (1989)

112. J.D. Scargle, Astrophys. J. 263, 835 (1982)

113. F.S. Ling, P. Sikivie, S. Wick, Phys. Rev. D 70, 123503 (2004)

114. C.M., Lederer, V.S., Shirley (eds.), Table of Isotopes, 7th edn. (Wiley, New York, 1978)

115. P. Belli et al., Nuovo Cimento A 101, 959 (1989)

116. M. Cribier et al., Astropart. Phys. 4, 23 (1995)

117. A. Rindi et al., LNGS report LNF-88/01(P) (1988)

118. F. Arneodo et al., Nuovo Cimento A 8, 819 (1999)

119. H. Wulandari et al., Astropart. Phys. 22, 313 (2004)

120. ICARUS Collaboration, internal report TM03-01

121. A. Borio di Tigliole et al., Europhys. Lett. 67, 1045 (2004)

122. M. Wojcik, Nucl. Instrum. Methods B 61, 8 (1991)

123. M. Ambrosio et al., Astropart. Phys. 7, 109 (1997)

124. M. Aglietta et al., Nuovo Cimento C 12, 467 (1987)

125. M. Aglietta et al., hep-ex/9905047

126. P. Belli et al., Phys. Rev. D 66, 043503 (2002)

127. R. Bernabei, DAMA Colloboration, A. Bottino, Nature 449, 24 (2007) 